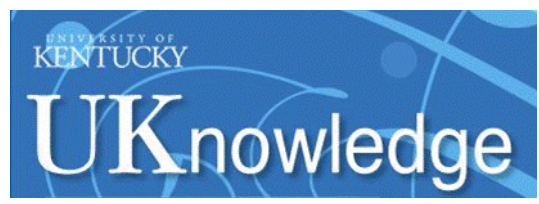

University of Kentucky

UKnowledge

\title{
Nebular Properties and the lonizing Radiation Field in the Galactic Center
}

Joseph C. Shields

The Ohio State University

Gary J. Ferland

University of Kentucky, gary@uky.edu

Follow this and additional works at: https://uknowledge.uky.edu/physastron_facpub

Part of the Astrophysics and Astronomy Commons, and the Physics Commons

Right click to open a feedback form in a new tab to let us know how this document benefits you.

\section{Repository Citation}

Shields, Joseph C. and Ferland, Gary J., "Nebular Properties and the lonizing Radiation Field in the Galactic Center" (1994). Physics and Astronomy Faculty Publications. 166.

https://uknowledge.uky.edu/physastron_facpub/166

This Article is brought to you for free and open access by the Physics and Astronomy at UKnowledge. It has been accepted for inclusion in Physics and Astronomy Faculty Publications by an authorized administrator of UKnowledge. For more information, please contact UKnowledge@lsv.uky.edu. 


\section{Nebular Properties and the Ionizing Radiation Field in the Galactic Center}

Digital Object Identifier (DOI)

http://dx.doi.org/10.1086/174398

\section{Notes/Citation Information}

Published in The Astrophysical Journal, v. 430, no. 1, p. 236-251.

(c) 1994. The American Astronomical Society. All rights reserved.

The copyright holder has granted permission for posting the article here. 
The Astrophysical Journal, 430:236-251, 1994 July 20

(C) 1994. The American Astronomical Society. All rights reserved. Printed in U.S.A.

\title{
NEBULAR PROPERTIES AND THE IONIZING RADIATION FIELD IN THE GALACTIC CENTER
}

\author{
JoSEPH C. SHIELdS ${ }^{1,2,3}$ AND GARY J. FERLAND ${ }^{2}$ \\ Received 1993 July 14 ; accepted 1994 January 20
}

\begin{abstract}
Nebulosity in the central parsec of the Milky Way exhibits a low-ionization spectrum that has led previous analyses of this region to conclude that this material is photoionized by a relatively soft continuum. We have reanalyzed the infrared emission-line spectrum of the Galactic center in order to consider whether the data could actually be explained with photoionization by a relatively hard, yet dilute continuum, and additionally to constrain the properties of the radiating plasma. We conclude that the composite infrared spectrum does not place strong restrictions on the nature of the ionizing continuum and that much of the ionized gas in the Galactic center is probably quite dense $\left(\gtrsim 10^{5} \mathrm{~cm}^{-3}\right)$. If the ionizing spectral energy distribution is, in fact, relatively hard, this material additionally must be highly clumped. Indications that the ionizing continuum is probably fairly soft are provided by radio recombination-line studies, however. Shocks are unlikely to be important for generating the observed nebulosity in light of the large far-infrared continuum luminosity of the central parsec. The infrared fine-structure spectrum provides mixed evidence for enhanced heavy-element abundances, and enrichment is probably limited to $\$ 2$ times solar levels. We predict the optical spectrum of the Galactic center and conclude that it probably appears as an $\mathrm{H}$ il nucleus to external observers. Comparisons between the nucleus of the Milky Way and nuclei of external galaxies remain uncertain, however, since the parsec-scale metric apertures typically employed for studies of Galactic-center nebular emission are 1-2 orders of magnitude smaller than those used in extragalactic measurements.
\end{abstract}

Subject headings: galaxies: nuclei - Galaxy: center - H II regions - infrared: ISM: lines and bands

\section{INTRODUCTION}

The center of the Milky Way exhibits a collection of unusual and poorly understood phenomena. For example, infrared imaging provides direct detections of massive stars that suggest substantial recent star formation within the central parsec (Krabbe et al. 1991, and references therein). The Galactic center also features unusual sources of nonthermal radio emission (e.g., Pedlar et al. 1989) and dynamical properties consistent with a large central mass concentration $\left(\sim 10^{6} M_{\odot}\right.$; e.g., Lacy et al. 1980; McGinn et al. 1989), inviting comparison with more luminous galactic nuclei that may be powered by accretion onto a black hole. Interest in the Galactic center thus remains strong, since it may be one of the most prolific starforming regions in the Milky Way, and possibly the nearest example of an active galactic nucleus (AGN). The fact that this region exhibits characteristics of both star formation and an AGN is itself noteworthy, since there are suggestions of evolutionary and/or causal connections between these phenomena (e.g., see the volume edited by Filippenko 1992).

The proximity of the Galactic center gives this region singular importance as a possible model for understanding the properties of other galaxy nuclei. Comparisons between the nucleus of the Milky Way and other galactic nuclei are complicated by this proximity, however. The large differences in projected spatial scale lead to difficulties in assigning correspondence between resolved structures in the Galactic center and the inferred constituents of extragalactic nuclei. In particular, synthesis of spectral energy distributions in a common metric aperture for the Galactic center and other nuclei remains challenging. The large extinction associated

\footnotetext{
${ }^{1}$ Department of Astronomy, Ohio State University, Columbus, OH 43210.

2 Department of Physics and Astronomy, University of Kentucky, Lexington, KY 40506.

${ }^{3}$ Current address: Steward Observatory, University of Arizona, Tucson, AZ 85721 .
}

with viewing the Galactic center through the plane of the Milky Way also eliminates the use of optical spectroscopic diagnostics that are commonly employed for the classification and characterization of emission-line nuclei (Baldwin, Phillips, \& Terlevich 1981; Veilleux \& Osterbrock 1987). Such distinctions based on optical spectra can suggest the extent to which the nuclear radiation field is generated by stars or an AGN over a broad range of intrinsic luminosity (Heckman 1980; Filippenko \& Sargent 1985).

Characterization of the ionizing radiation field of the Galactic center has consequently relied primarily on nebular emission at mid-infrared wavelengths. While infrared diagnostics analogous to optical line ratio methods have been proposed for nebular classification (Spinoglio \& Malkan 1992; Voit 1992), a dearth of comparison measurements leaves the efficacy of these diagnostics largely untested. The mid-IR spectrum of the central parsec of the Milky Way is characterized by emission from relatively low ionization species, leading Aitken et al. (1976), Lacy et al. (1980), and other researchers to suggest that the region is photoionized by stars with a characteristic temperature of $\lesssim 35,000 \mathrm{~K}$. While a very soft ionizing continuum will naturally lead to a low-ionization nebula, emission dominated by low-ionization species also arises from partially ionized gas irradiated by a dilute, hard continuum (Ferland \& Netzer 1983; Halpern \& Steiner 1983). Indeed, nuclei characterized by low-ionization optical emission are identified as LINERs (low-ionization nuclear emission-line regions; Heckman 1980) rather than $\mathrm{H}$ II nuclei, and strong evidence exists that some LINERs are photoionized by a very hard continuum (e.g., Filippenko \& Halpern 1984).

Ionization of the Galactic center nebula probably results from absorption of extreme-ultraviolet radiation with a spectral energy distribution that is of great interest but which remains unobservable directly. Within (large) metric apertures characteristic of extragalactic measurements, the X-ray lumi- 
nosity of the Galactic center approaches that of weak AGNs, with $L_{x} \approx 10^{37}-10^{38} \mathrm{ergs} \mathrm{s}^{-1}$ (Matteson 1982). The central few parsecs of the Milky Way contribute only a small fraction of this X-ray luminosity $\left(\sim 10^{35}\right.$ ergs $\mathrm{s}^{-1}$ within $0.9-4 \mathrm{keV}$; Watson et al. 1981), however, which is also small in comparison to the total ionizing luminosity within the same central zone ( $\sim 10^{50}$ ionizing photons $\mathrm{s}^{-1}$, implying $L_{\text {ion }}>10^{39}$ ergs $\mathrm{s}^{-1}$ ). If the central parsec-scale nebula is energized primarily by stars, it is nonetheless desirable to know the properties of the ionizing continuum and its specific sources. Terlevich \& Melnick (1985) have suggested that some LINERs may be generated through photoionization by extreme Wolf-Rayet stars. More recently, Filippenko \& Terlevich (1992) and Shields (1992) have found that relatively hot yet normal mainsequence $O$ stars might be responsible for generating many LINERs, with the distinction of these nebulae from $\mathrm{H}$ II regions traceable to high interstellar pressures and densities in galaxy nuclei. The role of hot, massive stars in energizing the Galactic center is thus of interest for assessing whether the Milky Way hosts a LINER, and more generally for defining the star formation history and initial mass function in the nucleus.

In order to constrain the properties of the ionizing radiation field as well as the physical conditions of the nebular gas in the Galactic center, we have compared the observed composite infrared spectrum of this region with emission-line intensities predicted for a range of ionizing continua and cloud properties. We present here the results of this comparison and discuss them in the context of independent observational constraints. We find that the emission-line spectrum is sensitive to physical properties other than simply the shape of the ionizing continuum, and it appears doubtful that the near-IR spectrum provides a compelling constraint on the ionizing spectral energy distribution. Radio recombination-line measurements probably offer the strongest evidence for a relatively soft ionizing continuum in this region. Detailed analysis of the observed spectrum suggests that much of the nebular gas in the Galactic center may be denser than previously assumed, and possibly highly clumped, with only modest heavy-element enrichment above solar levels.

The aims of the present work are similar to those of an earlier study by Cota (1987), who concluded that a soft radiation field was required to account for the composite infrared nebular spectrum of the central region. The differences between his conclusions and those presented here can be attributed largely to his use of single-component models for nebular analysis.

For those unfamiliar with the nomenclature of the Galactic center, we provide the following brief overview (see Genzel \& Townes 1987 for a comprehensive review). The region considered in this study consists of the central parsec of the Galaxy, which is identified with the parsec-scale nebula Sagittarius A West. In an AGN, this size scale would correspond to that of the inner narrow-line region (e.g., Osterbrock 1989). The ionized nebula is dominated by a filamentary, triskelion morphology, which has led to its identification as the "minispiral." The central portions of Sgr A West feature a collection of luminous stars known as IRS 16, and an anomalous compact radio source, $\mathrm{Sgr} \mathrm{A}^{*}$, that is often suggested as a candidate for a massive black hole. Additional infrared sources (presumably stars or groups of stars) are present in the vicinity and are identified by IRS number (e.g., IRS 1, IRS 7). The inner parsec of the Galaxy contains both ionized and neutral atomic gas, and is surrounded by a circumnuclear disk of dense and dusty molecular material. On large scales $(10-100 \mathrm{pc})$, the central region features unusual streamers of ionized gas perpendicular to the Galactic plane, and dense molecular clouds with embedded $\mathrm{H}$ II regions within the plane.

\section{EMPIRICAL AND THEORETICAL SPECTRA}

\subsection{Comparison Data}

For purposes of observational comparison, we derived a composite spectrum of the Galactic center from a compilation of published measurements. Table 1 lists the measured emission-line fluxes and corresponding apertures. This table is based in part on an earlier compilation by Cota (1987), which summarized the observations as they existed at that time. In order to construct a consistent set of fluxes, we scaled these values to a common aperture diameter of $30^{\prime \prime}$, corresponding to a linear size of $1.2 \mathrm{pc}$ for $R_{0}=8 \mathrm{kpc}$. This dimension represents a compromise between apertures ranging in size from a few arcseconds to an arcminute. Scale factors were derived from a growth curve for [Ne II] $12.8 \mu \mathrm{m}$ emission measured through varying beam sizes by Lacy et al. (1980) and extrapolated from their measurements to aperture diameters exceeding their maximum beam of $22^{\prime \prime}$. The resulting flux estimates for a common beam are listed for individual measurements in column (5) of Table 1.

Representing the composite spectrum by flux measurements rescaled in this manner is open to several obvious potential pitfalls. The degree to which the surface brightness distribution of the various lines follows that of $[\mathrm{Ne}$ II] is unknown. Aperture centering is known to differ between some measurements, with the indicated centers including IRS 1 , IRS 16 , and simply Sgr A West. The [Ne II] measurements used in deriving the growth curve were centered on IRS 1. High spatial-resolution maps of [Ne II] line emission from Sgr A West (Lacy, Achtermann, \& Serabyn 1991) suggest that nebular emission is sufficiently centrally concentrated that large-aperture measurements (beam size $\gtrsim 20^{\prime \prime}$ ) for emission that follows the [Ne II] distribution are unlikely to be seriously affected by such differences in aperture centering. We note additionally that rescaled fluxes for independent measurements of a given line in different beams generally show consistency to within a factor of 2. Rather poorer agreement is seen between the rescaled values for the Brackett lines, consistent with our expectation that small-aperture measurements may be subject to greater variation due to centering differences. These shortwavelength measurements are also in principle more sensitive to patchy variations in extinction.

Column (6) of Table 1 shows the resulting composite line fluxes for Sgr A West in a $30^{\prime \prime}$ beam. The tabulated values are simple averages of the measurements of the fluxes listed in column (5), with some exceptions. The strengths of the Brackett recombination lines are of particular importance for comparison with the strengths of the fine-structure emission, and for Bry we consequently base our final value on the measurement by Neugebauer et al. (1978), which was acquired through a relatively large beam of $32^{\prime \prime}$ diameter. This is close to our standard aperture size, and the corresponding standard flux is $\sim 20 \%$ higher than an average of scaled estimates from smaller beams. In a few other cases indicated in Table 1, listed measurements were selectively employed in order to improve agreement with the standard aperture, or maximize the restrictiveness of detection limits. 
TABLE 1

Galactic Center Emission-Line Measurements ${ }^{\mathrm{a}}$

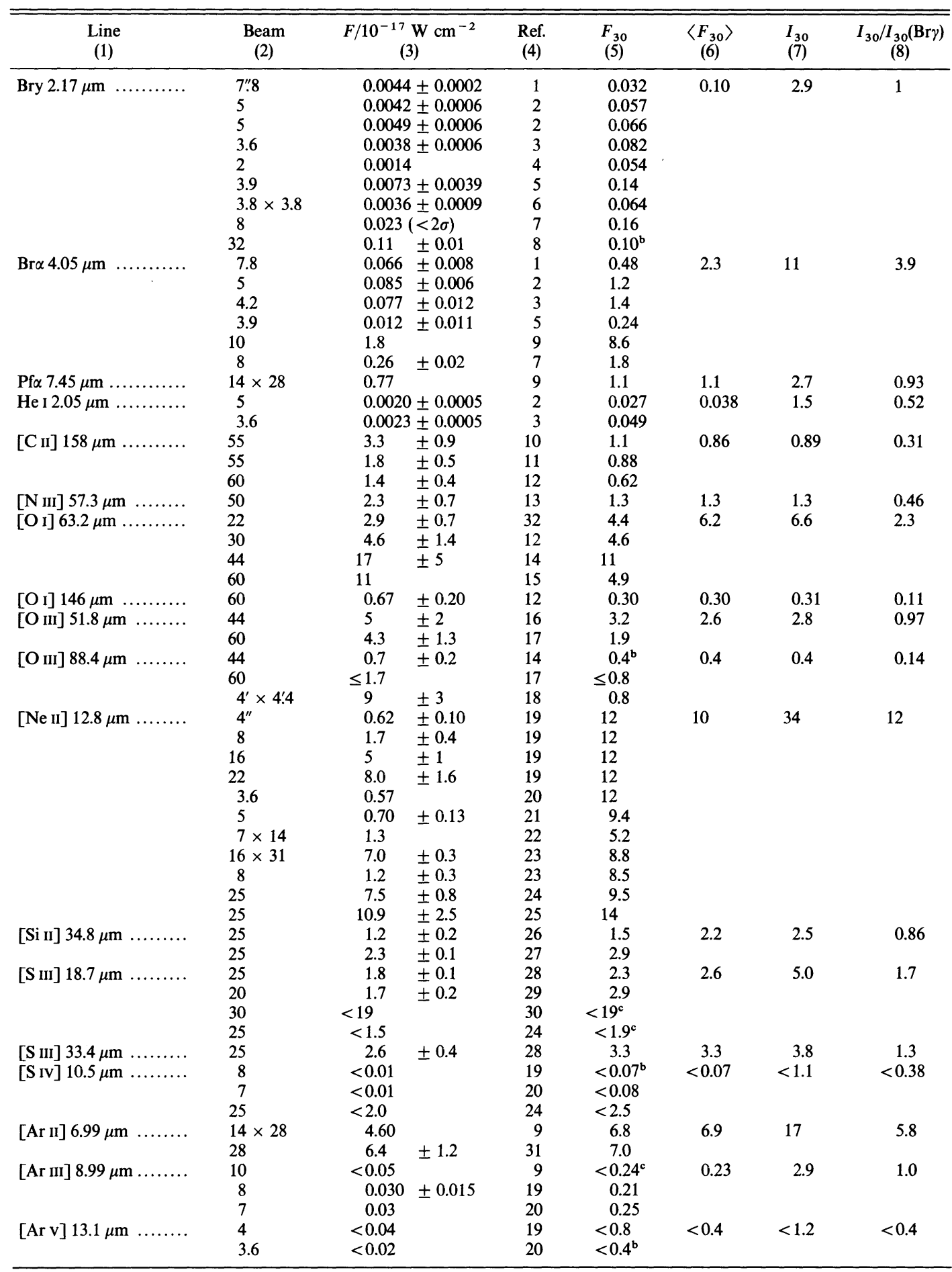

a Published emission-line measurements, where $F$ is flux in the indicated beam, "Ref." designates the reference listed below, $F_{30}$ is the measured flux corrected to an aperture diameter of $30^{\prime \prime},\left\langle F_{30}\right\rangle$ is the average $F_{30}, I_{30}$ is the same average after extinction correction, and $I_{30} / I_{30}(\mathrm{Br} \gamma)$ is the corrected flux normalized to that of $\mathrm{Br} \gamma$.

b Value adopted in place of an average.

c Value excluded from determining average.

REFERENCES.- (1) Rieke et al. 1989; (2) Wade et al. 1987; (3) Geballe et al. 1984; (4) Storey \& Allen 1983; (5) Willner \& Pipher 1983; (6) Wollman et al. 1982; (7) Bally et al. 1979; (8) Neugebauer et al. 1978; (9) Lester et al. 1981a; (10) Poglitsch et al. 1991; (11) Genzel et al. 1990;(12) Genzel et al. 1985;(13) Lester et al. 1987;(14) Genzel et al. 1984;(15) Lester et al. 1981b;(16) Watson et al. 1985; (17) Watson et al. 1980; (18) Dain et al. 1978; (19) Lacy et al. 1980; (20) Lacy et al. 1979; (21) Willner 1978; (22) Wollman et al. 1977; (23) Wollman et al. 1976; (24) Aitken et al. 1976; (25) Aitken et al. 1974; (26) Graf et al. 1988; (27) Herter et al. 1986; (28) Herter et al. 1984; (29) Herter et al. 1983; (30) McCarthy et al. 1980; (31) Willner et al. 1979; (32) Jackson et al. 1993. 
Estimating the intrinsic line spectrum of the Galactic center obviously requires correction for extinction. For this purpose, we use the extinction law given by Rieke, Rieke, \& Paul (1989) for 2-13 $\mu \mathrm{m}$, and that of Mathis (1990) with $R=3.1$ for longer wavelengths. The Rieke et al. curve is specific to the Galactic center and differs from the standard Mathis (1990) formulation within the same interval primarily by enhanced absorption in the $10 \mu \mathrm{m}$ feature. Extinction-corrected emission-line fluxes for our standard aperture are tabulated in column (7) of Table 1, and assume $A_{V}=31$ (Rieke et al. 1989). This final spectrum is employed for comparisons with predicted line intensities in our subsequent analysis.

In deriving this empirical composite spectrum, a number of rather uncertain corrections have been applied, and we hesitate to make any firm estimate of the uncertainties in the final values. Distinctions between theoretical possibilities on the basis of these values clearly must take into account the possible errors in the foregoing procedure; the most reliable comparisons are probably those that rest on ratios of lines measured in similar entrance apertures. The elimination of possible scenarios is more reliably based on disagreements in line ratios by an order of magnitude, rather than by factors of a few.

\subsection{Preliminary Considerations}

We performed a series of calculations using the photoionization code CLOUDY, version 84.00b (Ferland 1993), in an effort to reproduce the observed relative emission-line intensities. Previous observations provide variable degrees of constraint for initial parameter estimates in these calculations. Elemental abundances at the Galactic center remain illdetermined. We thus initially assumed solar abundances in our calculations (Grevesse \& Anders 1989). The strengths of the fine-structure lines are sensitive to heavy-element abundance, however, and we consequently ran additional test calculations in order to understand the influence of abundance modifications.

Gas-phase abundances are also sensitive to depletion onto grains. Estimates of the dust-to-gas ratio in the central parsec are consistent with extranuclear values (Davidson et al. 1992), although with large uncertainty reflecting choice of dust temperature and the degree to which tracers of atomic gas (such as [O I] emission) provide accurate estimates of gas mass. Depletion rates elsewhere in the Galaxy are known to be sensitive to environmental conditions (Cowie \& Songaila 1986). In AGNs, the sub-parsec-scale broad-line region appears to have a low dust content, presumably since grains cannot survive in this high radiation field environment (Netzer \& Laor 1993); the parsec- to kiloparsec-scale narrow-line region shows direct evidence for depletion (Ferland 1994), although the associated grain populations may be unusual (Laor \& Draine 1993). A recent study of the center of M31 similarly suggests that dust in this region may be peculiar (Lauer et al. 1993). To avoid reaching conclusions that are sensitive to specific choices of depletion rates within the Galactic center, we initially performed calculations assuming negligible depletion: Fortunately most of the elements observed in fine-structure emission are, in fact, expected to be only weakly influenced by depletion. We additionally computed test calculations with plausible amounts of interstellar dust and associated depeletion; the results and their implications are discussed in $\S 3.3$. In general, the sensitivity in predicted line strength to characteristics of the radiation field and nebular density is far greater than to depletion, and the influence of depletion propagates in a simple way into predictions of line strength.
In principle, the electron density $n_{e}$ in clouds in the central parsec can be constrained by observed line pairs of [S III], [O III], and [O I] (e.g., Rubin 1989). Assuming a uniform plasma, the $\left[\mathrm{S}\right.$ III] ratio suggests densities of a few times $10^{3}$ $\mathrm{cm}^{-3}$. Within the measurement uncertainties, the $[\mathrm{O} \mathrm{III}]$ ratio is consistent with this density, but permits larger values extending beyond its high density limit at $\sim 10^{5} \mathrm{~cm}^{-3}$ (Genzel et al. 1984). The [O I] ratio appears to reflect slightly higher $n_{e}$ than that indicated by [S III] and is consistent within measurement uncertainties with its high-density limit (Genzel et al. 1985). High densities $\left(n_{e}>10^{5} \mathrm{~cm}^{-3}\right)$ appear to be implicated by [Fe II $]$ emission-line ratios in a $\sim 5^{\prime \prime}$ aperture southwest of IRS 16, and similar measurements of [Fe III] lines (DePoy 1992 and private communication).

Discrepancies in density estimates from these diagnostics can arise from density and ionization structure in the nebula resulting in differing spatial emissivity distributions for the different species. This complication implies further that the existence of clouds with $n_{e}$ in excess of estimates from any of the nominal density diagnostics cannot be excluded. Cloud density is one determinant of the ionization parameter $U$, the dimensionless ratio of ionizing photon to nucleon density. Since the Galactic center exhibits evidence for prolific star formation and other energetic phenomena, the radiation density near the emission nebulosity may be relatively high; if the lowionization properties of the plasma reflect a very low $U$, clouds with quite high densities may, in fact, be indicated. We consequently examined possible emission contributions from nebular components exhibiting a wide range of density, rather than from a uniform medium with density fixed by one of the nominal density indicators.

CLOUDY treats levels $3 \leq n \leq 6$ of hydrogen assuming full mixing of $l$ states. In the physical conditions of interest here, $l$-mixing is likely to be incomplete. In order to specify a $\mathrm{Br} \gamma$ line intensity taking into account incomplete $l$-mixing, we used the predicted $\mathrm{H} \beta$ intensity, which is relatively insensitive to the $l$-state distribution, and scaled this line by the $\mathrm{Br} \gamma / \mathrm{H} \beta$ recombination ratio appropriate to the average electron temperature $T_{e}$ (calculated with weighting by the $\mathrm{H}^{+}$density) and average electron density. Case B recombination line ratios tabulated by Hummer \& Storey (1987) were used for this purpose.

Atomic helium is modeled as a 15 -level system. The helium singlets have 10 levels, with $1 s, 2$, and $3 p$ as in the actual atom, assuming that levels 3 through 6 are well $l$-mixed, and with three pseudo-levels representing levels 7 through 100 of the real atom. This approach is entirely analogous to that outlined by Ferland \& Rees (1988) for hydrogen. The triplets are treated with five levels. Collision data are taken from Berrington \& Kingston (1987). Radiative data are from standard sources (i.e., Osterbrock 1989; Wiese, Smith, \& Glennon 1966). Line transfer is treated using escape probabilities modified to include background opacities. For the Ly $\alpha$ transition of the helium singlets, interpolation on the Hummer \& Kunasz (1980) hydrogen Ly $\alpha$ results are used. For all other transitions Hummer's $K_{2}$ function (Hummer 1968, 1981) is used. Tests (Ferland 1993) show that the model atom goes to the interstellar medium case at low densities, and to LTE at high densities.

\subsection{Calculation Results}

\subsubsection{Photoionization by Cool O Stars}

Previous discussion of photoionization in the Galactic center has emphasized intensity ratios of [Ar II] $7 \mu \mathrm{m} /[\mathrm{Ar}$ III] $9 \mu \mathrm{m}$ (observed to be $\sim 6$ ) and [S III $] 18+33 \mu \mathrm{m} /[\mathrm{S}$ IV $] 10.5$ 
$\mu \mathrm{m}$ (observed to be $>7$ ) as diagnostics of the continuum spectral energy distribution (e.g., Lacy et al. 1980; Serabyn \& Lacy 1985; Cota 1987). Ionization of $\mathrm{Ar}^{+}$to produce $\mathrm{Ar}^{++}$requires a photon energy of at least $27.6 \mathrm{eV}$. Kurucz (1979) model stellar atmosphere continua show a $\mathrm{He}$ I absorption edge at $24.6 \mathrm{eV}$ that rapidly decreases in amplitude as stellar effective temperature $T_{*}$ is increased from $30,000 \mathrm{~K}$ to $40,000 \mathrm{~K}$. For normal $\mathrm{H}$ II regions, the dominant species of ionized argon consequently changes from $\mathrm{Ar}^{+}$to $\mathrm{Ar}^{++}$as $T_{*}$ is increased over this range. The $[\mathrm{Ar} \mathrm{II}] /[\mathrm{Ar} \mathrm{III}]$ ratio in particular is thus a sensitive indicator of $T_{*}$ for $\mathrm{H}$ II-region nebular conditions.

As a preliminary note of caution, however, we note that predicted emission by sulfur and argon is subject to uncertainty caused by a lack of reliable recombination rate coefficients. For second-row elements it is known that dielectronic recombination through low-lying autoionizing states is often the dominant recombination process at nebular temperatures (Nussbaumer \& Storey 1983). These rate coefficients have not been calculated for most third-row elements, and we use the mean of the second-row rate coefficients, as described by Ali et al. (1991). This procedure may introduce as much as a $50 \%$ error in the line ratio. Reliable calculations of the sulfur and argon ionization balance must await the completion of the extension to the Opacity Project described by Nahar \& Pradhan (1992).

Emission-line intensities resulting from a $35,000 \mathrm{~K}$, $\log (g)=4.5$, Kurucz model continuum incident on a cloud with hydrogen density $n_{\mathrm{H}}=10^{3.5} \mathrm{~cm}^{-3}$ are shown normalized to $\mathrm{Br} \gamma$ and as a function of ionization parameter $U$ in Figure 1. The nebula is assumed to be ionization-bounded (i.e., optically thick), and the calculation is stopped at a depth where $T_{e}$ falls below $1500 \mathrm{~K}$. Some additional emission from very low ionization species (C II, Si II, O I) may be generated at greater depths

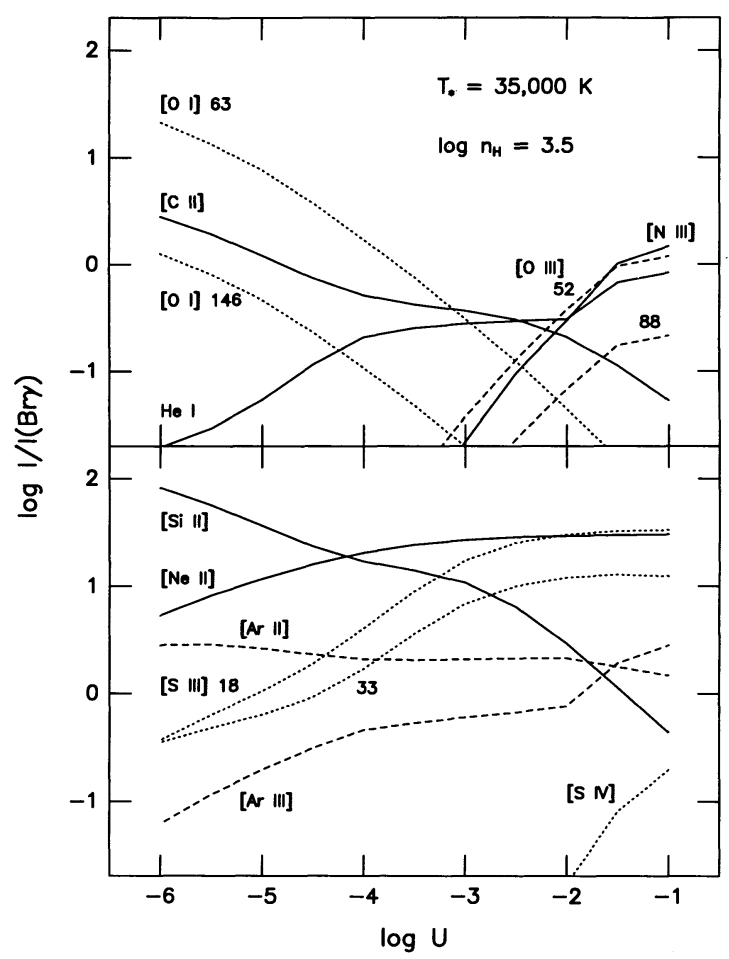

Fig. 1.-Predicted emission-line intensities normalized to $\mathrm{Br} \gamma$, as a function of $U$, for a cloud with $\log n_{\mathrm{H}}=3.5$ irradiated by a $35,000 \mathrm{~K}$ stellar continuum. and from photodissociation regions (PDRs; e.g., Tielens \& Hollenbach 1985; Wolfire, Tielens, \& Hollenbach 1990) at the surfaces of the molecular clouds surrounding the central parsec. The predicted flux in these lines should consequently be treated as lower limits. For the parameters used in the present calculations, emission in the fine-structure lines remained optically thin.

Figure 1 confirms the expectation that the intensity ratio [Ar II $] 7 \mu \mathrm{m} /[\mathrm{Ar}$ III $] 9 \mu \mathrm{m}$ is large so long as $U$ is not too high $(\log U \lesssim-2)$. For the same combination of parameters, emission in [S IV] $10.5 \mu \mathrm{m}$ is weak and [Ar v] $13.1 \mu \mathrm{m}$ is negligible, in agreement with the Galactic center measurements. The overall empirical spectrum is not well reproduced by the predictions in Figure 1 , however. For $-2 \gtrsim \log U \gtrsim-4$, the intensity ratio of $[\mathrm{S} \mathrm{III}] 18,33 \mu \mathrm{m} /[\mathrm{Ar}$ II $] 7 \mu \mathrm{m}$ is considerably larger than observed. The predicted emission in [Ne II 12.8 $\mu \mathrm{m}$ and [Si II] $35 \mu \mathrm{m}$ is also excessive, while the calculated [O III $]$ and [N III $]$ lines are weak. At lower $U,[\mathrm{Ne}$ II $]$ and [S III] emission is reduced to appropriate levels, but [Si II], [O I], and [C II] emission becomes much too strong, while $[\mathrm{O}$ III $]$ and $[\mathrm{N}$ III $]$ remain highly deficient.

A single component of gas with $n_{\mathrm{H}} \approx 10^{3.5} \mathrm{~cm}^{-3}$ ionized by a $35,000 \mathrm{~K}$ star appears incapable of generating line emission with the relative intensities observed in the Galactic center. Use of continua appropriate for stars of different temperature does not improve this situation. Additional calculations employing a range of stellar temperature demonstrate that [S III] and/or [S IV] emission tends to be too strong at large $U$; [O III] and [N $\mathrm{III}]$ is deficient except at large $U$, particularly as $T_{*}$ is reduced below $35,000 \mathrm{~K}$; and at low $U$, emission in $[\mathrm{Si} \mathrm{II}],[\mathrm{O} \mathrm{I}]$, and $[\mathrm{C} \mathrm{II}]$ is predicted to be much too strong. A single-component representation of the central nebula with $n_{\mathrm{H}} \approx 10^{3.5} \mathrm{~cm}^{-3}$ thus appears inadequate for generating the observed emission-line spectrum.

In reality, the ionized gas is probably characterized by significant structure on many scales, and the radiation field is likely to be spatially very inhomogeneous, requiring a description of the central nebula with multiple phases. A multicomponent system was already suggested by the differences in electron density $n_{e}$ indicated by independent nebular diagnostics ( $§ 2.2$; see also Genzel \& Townes 1987). The predicted line strengths shown in Figure 1 suggest that a component with density of order $10^{3} \mathrm{~cm}^{-3}$ and $\log U \approx-2$ could easily dominate emission in [S III], with other lines weak in contrast; additional components with correspondingly dominant emission in other lines would evidently be needed in order to give rise to the composite spectrum. One such component clearly missing from Figure 1 is one in which argon emission is strong relative to the other fine-structure lines.

The [Ar II] $7 \mu \mathrm{m}$ line is noteworthy for having a relatively high critical density, with $n_{\text {crit }} \approx 2 \times 10^{5} \mathrm{~cm}^{-3}$. This property suggests that a relatively dense component may be successful at generating strong emission from [Ar II] while collisionally suppressing emission in many of the other observed lines. Figure 2 shows the predicted line strengths as a function of $U$ as in Figure 1, but with $n_{\mathrm{H}}$ increased to $10^{5} \mathrm{~cm}^{-3}$. Emission in the [Ar II] line indeed exceeds that in the [S III] lines at this density, for $\log U \lesssim-3$. The lines of [Si II], [C II], and, to a lesser extent, $\left[\mathrm{O}_{\mathrm{I}}\right]$, are also reduced at a given $U$ relative to [Ar II]. Collisional de-excitation of the latter transitions results primarily from electron encounters for the physical conditions considered here, in contrast with PDRs in which neutral hydrogen tends to dominate collisional excitation processes 


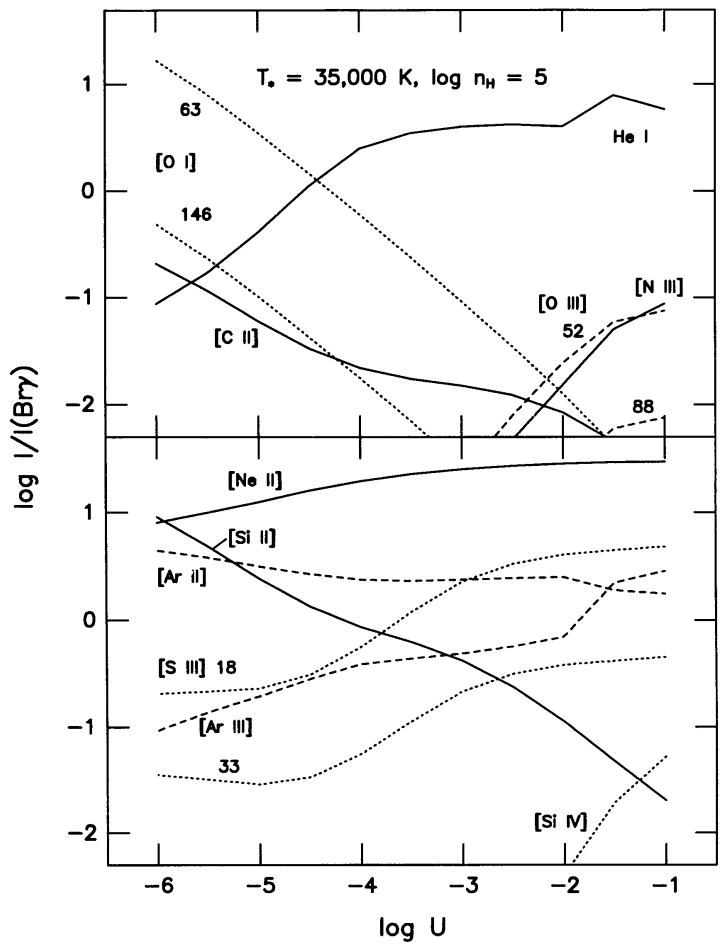

FIG. 2.-Predicted emission-line intensities normalized to $\mathrm{Br} \gamma$, as a function of $U$, for a cloud with $\log n_{\mathrm{H}}=5$ irradiated by a $35,000 \mathrm{~K}$ stellar continuum.

(Tielens \& Hollenbach 1985). The He I $2.06 \mu \mathrm{m}$ line is boosted significantly in the high-density case. Exchange collisions from the helium triplets to singlets proceed efficiently in this regime (Osterbrock 1989), leading to an increase in the singlet excitedstate population and associated emission.

Figures 1 and 2 do not indicate a clear means of obtaining emission in the lines of [O III] comparable to [S III], as observed. A cloud described by a low $n_{\mathrm{H}}$ and high $U$ may be one approach, although the density indicated by the [O III] $52 / 88 \mu \mathrm{m}$ ratio is relatively high $(\S 2.2)$ and at large $U$ the upper limit to [S IV] $10.5 \mu \mathrm{m}$ can become difficult to satisfy. One possible means of obtaining a strong [O III] component may be to relax our assumption that the emitting clouds are optically thick to the ionizing continuum. For an optically thick cloud, the $\mathrm{S}^{++}$zone extends to greater depth than the $\mathrm{O}^{++}$ zone; truncating the cloud so that the $\mathrm{S}^{++}$region is partially removed will tend to increase the $[\mathrm{O} \mathrm{III}] /[\mathrm{S}$ III $]$ intensity ratio.

As an illustration of how multiple nebular components with distinct properties could combine to yield the observed Galactic center spectrum, Table 2 lists the predicted line intensities for three components as discussed above, as well as a weighted composite. Optically thick clouds described by $\left(\log n_{\mathrm{H}}\right.$, $\log U)=(2.5,-2)$ and $(5,-4.8)$ and a cloud truncated at a column density of $10^{20} \mathrm{~cm}^{-2}$ with $(4,-0.5)$, chosen, respectively, to dominate emission in [S III], [Ar II], and [O III], were assumed to contribute $\mathrm{Br} \gamma$ luminosity in the ratio $0.03: 1: 0.12$. In all cases the ionizing continuum was again assumed to be that of a $35,000 \mathrm{~K}$ star. Despite the simplicity of this model and the significant observational uncertainties, the composite prediction of relative line strengths reproduces many important aspects of the Galactic center spectrum. In particular, the relative strengths of the $[\mathrm{S} \mathrm{III]},[\mathrm{Ar}$ III $]$, and [O III] lines are approximately correct, and the nominal density indicated by the [S III] $18 / 33 \mu \mathrm{m}$ ratio is less than that implied by [O III] $52 / 88 \mu \mathrm{m}$ ( $\sim 2000 \mathrm{~cm}^{-3}$ vs. $\sim 6000 \mathrm{~cm}^{-3}$; Rubin 1989), as observed.

Several features of the empirical spectrum are not reproduced in detail by the composite prediction, but none of these discrepancies appears serious. Predicted emission in [C II] is less than observed, consistent with the possibility that additional PDR contributions may be present in the large beam sizes employed for measurement of this line. Another lowionization feature, $[\mathrm{Si}$ II $]$, is predicted to be stronger than observed by roughly a factor of 2 . The two values can be easily reconciled if silicon is slightly depleted onto grains. Generation

TABLE 2

35,000 K Stellar Photolonization Predictions

\begin{tabular}{|c|c|c|c|c|c|}
\hline \multirow[b]{2}{*}{ PARAMETER } & \multicolumn{3}{|c|}{ COMPONENT NUMBER } & \multirow[b]{2}{*}{ CoMposite } & \multirow[b]{2}{*}{ OBSER VED } \\
\hline & 1 & $2^{\mathrm{a}}$ & 3 & & \\
\hline (1) & 2.5 & 4 & 5 & & \\
\hline 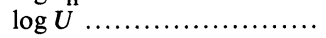 & -2 & -0.5 & -4.8 & & \\
\hline Weight ................... & 0.03 & 0.12 & 1 & & \\
\hline \multicolumn{6}{|l|}{ Relative Line Intensities: } \\
\hline $\mathrm{Br} \gamma 2.17 \mu \mathrm{m}$ & 1 & 1 & 1 & 1 & 1 \\
\hline $\mathrm{He}$ I $2.05 \mu \mathrm{m} \ldots \ldots \ldots \ldots \ldots$ & 0.16 & 0.16 & 0.64 & 0.56 & 0.52 \\
\hline$[\mathrm{C}$ II $] 158 \mu \mathrm{m} \ldots \ldots \ldots \ldots$ & 1.8 & $6.4 \times 10^{-4}$ & 0.047 & 0.089 & 0.31 \\
\hline$\left[\mathrm{N}_{\mathrm{III}}\right] 57.3 \mu \mathrm{m} \ldots \ldots \ldots \ldots$ & 1.2 & 0.90 & $6.0 \times 10^{-6}$ & 0.13 & 0.46 \\
\hline$\left[\mathrm{O}_{\mathrm{I}}\right] 63.2 \mu \mathrm{m} \ldots \ldots \ldots \ldots$ & 0.046 & $4.5 \times 10^{-6}$ & 2.5 & 2.2 & 2.3 \\
\hline$[\mathrm{O}$ I $] 146 \mu \mathrm{m} \ldots \ldots \ldots \ldots$ & $4.1 \times 10^{-3}$ & $2.2 \times 10^{-7}$ & 0.072 & 0.063 & 0.11 \\
\hline [O III] $51.8 \mu \mathrm{m} \ldots \ldots \ldots \ldots$ & 0.81 & 8.8 & $8.7 \times 10^{-6}$ & 0.94 & 0.97 \\
\hline$[\mathrm{O}$ III $] 88.4 \mu \mathrm{m} \ldots \ldots \ldots \ldots$ & 0.54 & 1.1 & $9.1 \times 10^{-7}$ & 0.13 & 0.14 \\
\hline$[\mathrm{Ne}$ II $] 12.8 \mu \mathrm{m} \ldots \ldots \ldots \ldots$ & 28 & 12 & 14 & 14 & 12 \\
\hline$[\mathrm{Si} \mathrm{II}] 34.8 \mu \mathrm{m} \ldots \ldots \ldots \ldots$ & 13 & $3.4 \times 10^{-3}$ & 1.9 & 2.0 & 0.86 \\
\hline$[\mathrm{S}$ III $] 18.7 \mu \mathrm{m} \ldots \ldots \ldots \ldots$ & 30 & 9.3 & 0.25 & 2.0 & 1.7 \\
\hline [S III $] 33.4 \mu \mathrm{m} \ldots \ldots \ldots \ldots$ & 40 & 1.8 & 0.029 & 1.3 & 1.3 \\
\hline [S IV $] 10.5 \mu \mathrm{m} \ldots \ldots \ldots \ldots$ & 0.016 & 2.2 & 0 & 0.23 & $<0.38$ \\
\hline$[\mathrm{Ar} \mathrm{II}] 6.99 \mu \mathrm{m} \ldots \ldots \ldots \ldots$ & 2.0 & 0.043 & 2.9 & 2.6 & 5.8 \\
\hline$[\mathrm{Ar}$ III $] 8.99 \mu \mathrm{m} \ldots \ldots \ldots$ & 0.66 & 3.0 & 0.23 & 0.53 & 1.0 \\
\hline$[$ Ar v $] 13.1 \mu \mathrm{m} \ldots \ldots \ldots . . .$. & 0 & 0 & 0 & 0 & $<0.4$ \\
\hline
\end{tabular}

a Truncated at hydrogen column density of $10^{20} \mathrm{~cm}^{-2}$. 
of additional [C $\mathrm{II}]$ emission that is not accompanied by excessive $[\mathrm{O} \mathrm{I}]$ or $[\mathrm{Si}$ II] emission can result from a dusty PDR that is clumped with a range of density (Meixner \& Tielens 1993).

The strength of $[\mathrm{N} \mathrm{III]}$ is underpredicted by nearly a factor of 4. This feature can be enhanced to the observed level by increasing the column density of the high- $U$ component, but at the expense of generating excessive $[\mathrm{S}$ III] emission from the same material. On theoretical and empirical grounds, nitrogen enrichment is expected to scale approximately as the square of global metallicity, so that agreement between observations and predictions of the $[\mathrm{N}$ III] line may be particularly sensitive to assumptions concerning abundances (see $\S 3.3$ ). Emission in both argon lines is underpredicted by a factor of $\sim 2$. This disagreement is not readily removed by adjustment of nebular parameters. While the discrepancy may be within the uncertainties of the empirical spectrum, the weakness of predicted [Ar II], [Ar III], and [N III] emission may provide evidence for heavy-element enrichment above solar levels, a point considered further in $\S 3.3$.

The strong emission in [Ar II] $7 \mu \mathrm{m}$ compared to other lines apparently necessitates that much of the emitting plasma be relatively dense $\left(n_{\mathrm{H}} \gtrsim 10^{5} \mathrm{~cm}^{-3}\right)$. The present model assumes that this phase is also described by an ionization parameter $(\log U=-4.8)$ that is considerably lower than that describing most material in typical $H$ II regions $(\log U \approx-2.5)$. The low $U$ in the present model is dictated primarily by the behavior of the $\mathrm{He}$ I $2.06 \mu \mathrm{m}$ line. (The $[\mathrm{S} \mathrm{III}] /[\mathrm{Ar}$ II $]$ ratio can be reduced by adopting a slightly higher $n_{\mathrm{H}}$.) At higher $U, \mathrm{He} \mathrm{I} / \mathrm{Br} \gamma$ is predicted to be much stronger than the observed value of $\sim 0.5$. As noted previously, He I $2.06 \mu \mathrm{m}$ can be strongly enhanced at high densities when exchange collisions become significant. However, the strength of this feature is sensitive to competition between helium scattering and hydrogen and dust absorption of the He I $\lambda 584$ line (Osterbrock 1989). For higher $U$, the He I ionization front is more sharply defined (Davidson $\&$ Netzer 1979), resulting in a region near the front in which helium is partially ionized while hydrogen remains highly ionized. This behavior facilitates efficient production and scattering of $\lambda 584$ resulting in resonance fluorescence of the 2.06 $\mu \mathrm{m}$ feature. At lower $U$ the helium and hydrogen $I$-fronts are both extended over a large fraction of the nominally ionized zone, and the elevated hydrogen neutral fraction in the region of recombining helium leads to increased destruction of the $\lambda 584$ photons and diminution of $2.06 \mu \mathrm{m}$ emission.

If $n_{\mathrm{H}}$ is indeed high, the observed $\mathrm{He} \mathrm{I} / \mathrm{Br} \gamma$ ratio suggests that this material is described by a low $U$, a property that may follow naturally from the relationship between density and $U$. One means of reducing the strength of $\mathrm{He}$ I $2.06 \mu \mathrm{m}$ emission at higher $U$ is by increasing the dust-to-gas ratio, which could harden the ionizing continuum (see $\S 3.3$ ) and thus reduce the neutral He fraction capable of scattering $\lambda 584$ photons, while also increasing the density of grains capable of destroying $\lambda 584$ photons. Test calculations assuming an interstellar graphite/ silicate grain population indicate that elevating the dust content by plausible factors is unlikely to play a large role in reducing $2.06 \mu \mathrm{m}$ emission. In fact, for cool stellar continua, the tendency for dust to harden the continuum will more likely increase the strength of $2.06 \mu \mathrm{m}$ emission by increasing the total volume of recombining helium within the region of ionized hydrogen.

A more promising mechanism for diminishing the $2.06 \mu \mathrm{m}$ line is by addition of turbulence on small scales, which will tend to increase the mean free path between scatterings of the $\lambda 584$ resonance line and hence the likelihood per scattering that these line photons will be absorbed by hydrogen or dust. Calculations with $n_{\mathrm{H}}=10^{5} \mathrm{~cm}^{-3}$ and $U=0.01$ predict $\mathrm{He} \mathrm{I}$ $2.06 \mu \mathrm{m} / \mathrm{Br} \gamma$ of 4.0 for thermal velocities, which is reduced to 1.8 with the addition of $50 \mathrm{~km} \mathrm{~s}^{-1}$ microturbulence (velocity full width at half-maximum). Turbulent velocities as high as this figure would be consistent with measured line widths (Roberts \& Goss 1993), and have little effect on photoionization predictions for the other measured lines. The reduction obtained with turbulence still leaves the predicted $\mathrm{He} \mathrm{I} / \mathrm{Br} \gamma$ ratio at $U=0.01$ more than 3 times that observed in the central parsec. It should be emphasized, however, that the strength of the He I $2.06 \mu \mathrm{m}$ line is highly sensitive in general to detailed nebular properties and geometry (Shields 1993). Although a model with predominantly high $n_{\mathbf{H}}$ and $U$ is not directly supported by the present calculations, it may be premature to exclude such a scenario based on the $\mathrm{He}$ I feature alone.

The results described in this section indicate that the emission-line spectrum of the Galactic center can be plausibly accounted for by photoionization by a radiation field generated by stars with $T_{*}=35,000 \mathrm{~K}$, as commonly assumed. Test calculations imply that the observed emission in [O III], [Ar III] and possibly other lines may be hard to reproduce with stars significantly cooler than this temperature, and we conclude that $T_{*} \gtrsim 30,000 \mathrm{~K}$. A uniform cloud irradiated by a $35,000 \mathrm{~K}$ stellar continuum does not adequately reproduce the complete spectrum, however. We emphasize that the threecomponent model discussed in this section is without doubt an extreme simplification of nebular structure in the Galactic center, and this representation should not be taken as a unique description of the emitting plasma. The fact that the predicted spectrum responds so strongly to the assumed nebular properties suggests all the more that a review of established conclusions concerning the nature of the ionizing radiation field is in order.

\subsubsection{Photoionization by Harder Continua}

The results of the previous section imply that a significant part of the Galactic center nebula may be described by a lower $U$ and higher $n_{\mathrm{H}}$ than is found in typical $\mathrm{H}$ II regions, even if the ionizing sources are relatively late-type $O$ stars. If the radiation field is actually significantly harder, the low $U$, high $n_{\mathrm{H}}$ component is likely to be even more essential for producing the observed spectrum. In order to assess the possibility that the Galactic center ionizing continuum is relatively hard, we thus initially examined the consequences of embedding optically thick clouds of variable $n_{\mathrm{H}}$ in a fixed radiation field described by a $50,000 \mathrm{~K}, \log (g)=4.5$, Kurucz model, such that increasing $n_{\mathrm{H}}$ resulted in corresponding decreases in $U$. The results are shown in Figure 3, where the ionizing radiation field has been fixed to yield consistency with $\mathrm{H}$ II regions at low densities (i.e., $\log U \approx-2.5$ for $\log n_{\mathrm{H}} \approx 3$ ). For nebular conditions appropriate for normal $\mathrm{H}$ II regions, the predicted [Ar II]/[Ar III] ratio is less than 0.1 , which is clearly inconsistent with the Galactic center observations. This behavior underlies the contention that such hot stars cannot be responsible for generating the Galactic center nebulosity. Increasing the cloud density changes this pattern, however, As $n_{\mathrm{H}}$ is increased and $U$ is correspondingly reduced, the $[\mathrm{Ar} \mathrm{II}] /[\mathrm{Ar} \mathrm{III}]$ ratio increases until it achieves consistency with the Galactic center measurement at $\left(\log n_{\mathrm{H}}, \log U\right) \approx(6.5,-6)$.

The results shown in Figure 3 indicate that a hard ionizing 


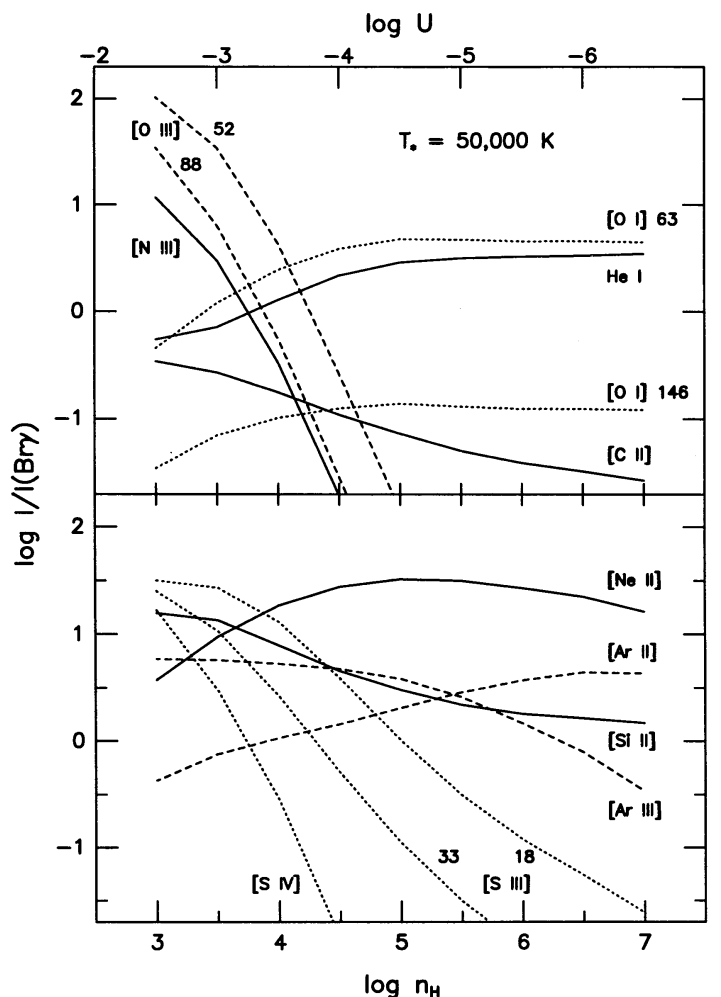

Fig. 3.-Predicted emission-line intensities normalized to $\mathrm{Br} \gamma$, for clouds in a fixed radiation field described by a $50,000 \mathrm{~K}$ stellar continuum. The ionizing photon density is set so that $\log U=0.5-\log n_{\mathrm{H}}$

continuum should not be dismissed out of hand for the Galactic center, although, as for the nebula photoionized by the $35,000 \mathrm{~K}$ star, a single $\left(n_{\mathrm{H}}, U\right)$ component is clearly inadequate for producing the overall spectrum. We consequently attempted to construct a simple three-component representation of the emitting nebula with the prescription employed in $\S 2.3 .1$, independently maximizing emission in [S III], [Ar II], and [O III].
The result, tabulated in Table 3 , assumes optically thick clouds with $\left(\log n_{\mathrm{H}}, \log U\right)=(3,-3.5),(7,-6),(4,-2.2)$, radiating $\mathrm{Br} \gamma$ in the proportions 0.05:1:0.02.

The agreement with the observations is comparable to that found for the softer radiation field in many aspects. Notable discrepancies are again found for [N III] and [Si II]. The [Ar II $] /[\mathrm{Ar}$ III $]$ ratio is in reasonable agreement with the observed value, but, as for the softer radiation field, the strength of both argon lines is somewhat weak relative to $\mathrm{Br} \gamma$. The contrast between the [S III] and [O III] densities is modest, reflecting a reduced degree of independence of the two lines. For the harder radiation field, the $\mathrm{S}^{++}$zone is not appreciably larger than the $\mathrm{O}^{++}$zone, so that truncating the cloud does not significantly increase the $[\mathrm{O} \mathrm{III}] /[\mathrm{S} \mathrm{III}]$ ratio. In addition, at $U$ sufficient to generate strong [O III] emission, emission in [S III] also tends to be strong or is supplanted by [S IV] emission. In Table 3, predicted emission in [S IV] is slightly in excess of the detection upper limit, although by an amount that is probably not significant.

Though the $50,000 \mathrm{~K}$ stellar photoionization model is reasonably successful at replicating the forbidden-line spectrum of the Galactic center, predicted emission of $\mathrm{He}$ I $2.06 \mu \mathrm{m}$ strongly exceeds that observed. The large predicted strength of this feature is again traceable primarily to the enhancement of the helium singlet states by exchange collisions from the triplets at high $n_{e}$. Unlike the predictions for the $35,000 \mathrm{~K}$ stellar continuum, $\mathrm{He}$ I $2.06 \mu \mathrm{m}$ in the present scenario is not highly sensitive to $U$ in the low- $U$ regime. Nebulae photoionized by stars with $T_{*} \gtrsim 40,000 \mathrm{~K}$ do not have a He interior to the $\mathrm{H} I$-front/ $I$-front. The spatial ionization structure of the two elements consequently evolves more nearly in parallel as a function of $U$ for the $50,000 \mathrm{~K}$ continuum than for the $35,000 \mathrm{~K}$ continuum. However, as noted earlier $(\S 2.3 .1)$, emission in He I $2.06 \mu \mathrm{m}$ will be reduced if significant small-scale velocity structure is present within the nebula. Test calculations indicate that addition of $30 \mathrm{~km} \mathrm{~s}^{-1}$ microturbulence results in a reduction of $\mathrm{He} \mathrm{I} / \mathrm{Br} \gamma$ to 0.65 , which is in reasonable agreement with the observed ratio.

TABLE 3

50,000 K Stellar Photolonization Predictions

\begin{tabular}{|c|c|c|c|c|c|}
\hline \multirow[b]{2}{*}{ PARAMETER } & \multicolumn{3}{|c|}{ COMPONENT NUMBER } & \multirow[b]{2}{*}{ CoMPOSITE } & \multirow[b]{2}{*}{ OBSERVED } \\
\hline & 1 & 2 & 3 & & \\
\hline $\log n_{H} \ldots \ldots \ldots \ldots \ldots$ & 3 & 4 & 7 & & \\
\hline 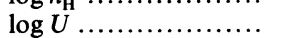 & -3.5 & -2.2 & -6 & & \\
\hline Weight ................... & 0.05 & 0.02 & 1 & & \\
\hline \multicolumn{6}{|l|}{ Relative Line Intensities: } \\
\hline $\mathrm{Br} \gamma 2.17 \mu \mathrm{m}$ & 1 & 1 & 1 & 1 & 1 \\
\hline He I $2.05 \mu \mathrm{m} \ldots \ldots \ldots$ & 0.50 & 1.2 & 3.6 & 3.4 & 0.52 \\
\hline$\left[\mathrm{C}_{\mathrm{II}}\right] 158 \mu \mathrm{m} \ldots \ldots$ & 1.5 & 0.022 & 0.014 & 0.084 & 0.31 \\
\hline$\left[\mathrm{N}_{\text {III }}\right] 57.3 \mu \mathrm{m} \ldots \ldots$ & 2.2 & 2.0 & $4.0 \times 10^{-7}$ & 0.14 & 0.46 \\
\hline$\left[\mathrm{O}_{1}\right] 63.2 \mu \mathrm{m} \ldots \ldots$ & 2.9 & 0.20 & 1.9 & 1.9 & 2.3 \\
\hline$\left[\mathrm{O}_{\mathrm{I}}\right] 146 \mu \mathrm{m} \ldots \ldots \ldots$ & 0.22 & $8.1 \times 10^{-3}$ & 0.052 & 0.059 & 0.11 \\
\hline [O III $] 51.8 \mu \mathrm{m} \ldots \ldots$ & 18 & 28 & $4.1 \times 10^{-6}$ & 1.4 & 0.97 \\
\hline$[\mathrm{O}$ III $] 88.4 \mu \mathrm{m} \ldots \ldots$ & 6.3 & 3.6 & $4.1 \times 10^{-7}$ & 0.36 & 0.14 \\
\hline$[\mathrm{Ne}$ II $] 12.8 \mu \mathrm{m} \ldots \ldots$ & 18 & 2.0 & 12 & 12 & 12 \\
\hline [Si II] $34.8 \mu \mathrm{m} \ldots \ldots$ & 44 & 1.4 & 0.68 & 2.7 & 0.86 \\
\hline [S III] $18.7 \mu \mathrm{m} \ldots \ldots$ & 21 & 17 & 0.019 & 1.3 & 1.7 \\
\hline [S III] $33.4 \mu \mathrm{m} \ldots \ldots$ & 17 & 3.4 & $1.7 \times 10^{-3}$ & 0.88 & 1.3 \\
\hline [S IV] $10.5 \mu \mathrm{m} \ldots \ldots$ & 0.37 & 26 & 0 & 0.50 & $<0.38$ \\
\hline [Ar II $76.99 \mu \mathrm{m} . . . . .$. & 1.0 & 0.28 & 2.8 & 2.7 & 5.8 \\
\hline [Ar III] $8.99 \mu \mathrm{m} \mathrm{.....}$ & 5.2 & 5.9 & 0.34 & 0.67 & 1.0 \\
\hline$[\mathrm{Ar} \mathrm{v}] 13.1 \mu \mathrm{m} \ldots \ldots$ & 0 & 0 & 0 & 0 & $<0.4$ \\
\hline
\end{tabular}


The results of this section demonstrate that the relative strengths of the infrared lines measured in the Galactic center do not, in themselves, strongly restrict the spectral energy distribution of the ionizing continuum in this region. Terlevich \& Melnick (1985) suggest that a relatively exotic population of WO and WC stars could yield a power-law continuum extending through the extreme-ultraviolet region, which could produce apparent AGN emission from surrounding gas; test calculations for such a continuum suggest that this quite hard spectral energy distribution also cannot be excluded on the basis of the mid-infrared spectrum. As discussed in $\S 3$, independent diagnostics for the properties of the Galactic center plasma and its ionization mechanisms exist, and to varying degrees offer constraints beyond those provided by the finestructure lines.

\subsection{Shocks as an Alternative Ionization Mechanism}

Shocks provide an alternative means of producing gas emitting a low-ionization forbidden line spectrum (Shull \& McKee 1979), and in the extragalactic environment, discrimination between shocks and photoionization for generation of LINERs continues to be an active topic of discussion (e.g., Ho, Filippenko, \& Sargent 1993). Shocks were previously considered by Lacy et al. (1980) as a possible ionizing mechanism within the Galactic center, but rejected by them in part because the predicted surface brightness of line emission from such a model was low by orders of magnitude compared to observed values. It should be noted, however, that this conclusion is dependent on assumptions concerning density of the shocking medium.

The kinetic energy flux at the shock face and hence the emitted post-shock radiative flux will scale with the density of the preshock medium. For a shock moving at $100 \mathrm{~km} \mathrm{~s}^{-1}$ into a medium with $n_{\mathrm{H}}=100 \mathrm{~cm}^{-3}$, Shull \& McKee (1979) list a predicted intensity of emitted $\mathrm{H} \beta$ as $3.31 \times 10^{-5} \mathrm{ergs} \mathrm{cm}^{-2}$ $\mathrm{s}^{-1} \mathrm{sr}^{-1}$, which implies an emitted $\mathrm{Br} \gamma$ intensity of approximately $1 \times 10^{-6}$ ergs $\mathrm{cm}^{-2} \mathrm{~s}^{-1} \mathrm{sr}^{-1}$. The observed, extinction-corrected $\mathrm{Br} \gamma$ intensity in our standard Galactic center aperture is $1.8 \times 10^{-2} \mathrm{ergs} \mathrm{cm}^{-2} \mathrm{~s}^{-1} \mathrm{sr}^{-1}$. If we assume a preshock $n_{\mathrm{H}}=10^{3}-10^{4} \mathrm{~cm}^{-3}$, and scale the predicted $\mathrm{Br} \gamma$ intensity by a corresponding factor of $10-100$, the predicted $\mathrm{Br} \gamma$ surface brightness is indeed lower than observed by 2-3 orders of magnitude. However, if densities within the central parsec are as high as $10^{6} \mathrm{~cm}^{-3}$, as discussed elsewhere in this paper, the original $\mathrm{Br} \gamma$ prediction should be scaled by a factor of order $10^{4}$, yielding $\sim 1 \times 10^{-2}$ ergs $\mathrm{cm}^{-2} \mathrm{sr}^{-1}$, in approximate agreement with the observed intensity. The shock velocity largely determines the degree of ionization of the postshock material, and a velocity of order $100 \mathrm{~km} \mathrm{~s}^{-1}$ is appropriate for generation of an optical LINER-like spectrum. Nebular components with $n_{\mathrm{H}} \gtrsim 10^{6} \mathrm{~cm}^{-3}$ are also present in at least some LINERs (Filippenko \& Halpern 1984).

The foregoing comparison suggests that the possibility of substantial shock ionization within the central parsec should be reconsidered. Shocks would tend to produce nebular components with $T_{e}$ in excess of that expected from photoionization. An analysis of this issue in the context of the Galactic center by Maloney, Hollenbach, \& Townes (1992) suggests, however, that emission from a hot phase of this type would probably be swamped by radiation from associated cooler phases. Hollenbach \& McKee (1989) have computed shock models with preshock densities as high as $10^{6} \mathrm{~cm}^{-3}$. Their calculations include predictions for the lines of $[\mathrm{C} \mathrm{II}],[\mathrm{O} \mathrm{I}]$, and $[\mathrm{Ne} \mathrm{II}]$ observed in the Galactic center. At a shock velocity of $\sim 100 \mathrm{~km} \mathrm{~s}^{-1}$, their predicted surface brightnesses for the [O I] lines are in good agreement with those observed in the central parsec, but the predicted strength of [C II] and [Ne II] falls short of the observed surface brightness by approximately an order of magnitude. Shock ionization thus does not appear to satisfactorily explain the observed line ratios in the central parsec unless Sgr A West is described by shocks that differ in some structural way from the models employed by Hollenbach \& McKee (1989), e.g., by inclination effects, a range of cloud densities and shock velocities, and additional ionization caused by embedded stars, so as to modify the observed spectrum from their prediction.

Probably the strongest evidence indicative of photoionization rather than shocks within the central parsec comes from the luminosity in the infrared continuum relative to line emission. For photoionized material, the infrared luminosity radiated by heated grains is expected to be an order of magnitude larger than that produced in shocks generating the same total infrared line luminosity (Hollenbach \& McKee 1989). The infrared continuum luminosity of Sgr A West is $\sim 2 \times 10^{6} L_{\odot}$ (Davidson et al. 1992), which is in good agreement with $\mathrm{H}$ II regions elsewhere (Wynn-Williams \& Becklin 1974) that undergo the same rate of ionization $\left(10^{50} \mathrm{~s}^{-1}\right.$; Lacy et al. 1980). Expressed in another form, $L_{\mathrm{IR}} / L(\mathrm{Br} \gamma) \approx 4 \times 10^{3}$ for the Galactic center. For shocks with velocity of $100 \mathrm{~km} \mathrm{~s}^{-1}$ moving into media with a density of $10^{6} \mathrm{~cm}^{-3}$, Hollenbach \& McKee (1989) estimate that associated grains will radiate with an intensity of $\sim 1$ ergs $\mathrm{cm}^{-2} \mathrm{~s}^{-1} \mathrm{sr}^{-1}$ for a standard dust-togas ratio. Combining this value with the $\mathrm{Br} \gamma$ flux of $\sim 10^{-2}$ ergs $\mathrm{cm}^{-2} \mathrm{~s}^{-1}$ estimated above yields a prediction of $L_{\mathrm{IR}} / L(\mathrm{Br} \gamma) \approx 100$ for shock-ionized plasma, which is more than an order of magnitude less than observed.

Photoionization thus appears to be far more likely as the process dominating the ionization of the central parsec unless other phenomena influence the rate of dust heating. The old stellar population could conceivably be important in this regard. Ultraviolet spectra of early-type galaxies commonly show a rise shortward of $2000 \AA$ that correlates with metallicity and is thought to arise from a population of evolved, low-mass stars (e.g., Burstein et al. 1988; Greggio \& Renzini 1990). The bulge of the Milky Way, which is old and relatively metal-rich (e.g., Rich 1988), probably contains a similar population. Studies of external galaxies imply that these stars have $T_{*} \lesssim$ 30,000 K (Ferguson et al. 1991; Ferguson \& Davidsen 1993). The persistence of a strong stellar density gradient in the Milky Way bulge to small radii suggests that such stars will be numerous within the central parsec (Kent 1992, and references therein). Due to their intermediate temperature, such stars would likely contribute negligibly to the nebular ionization, but would produce radiation just longward of the Lyman limit that will contribute to grain heating. The importance of grain heating by the old stellar population is evident in observational studies of $\mathrm{E}$ and $\mathrm{S} 0$ galaxies that show $L_{\mathrm{IR}} / L(\mathrm{H} \alpha)$ to be larger by a factor of 10 or more compared with the same ratio in star-forming disks or $\mathrm{H}$ II regions [Shields 1991; $L_{\mathrm{IR}} / L(\mathrm{H} \alpha) \approx 10^{3}$ in the early-type systems, implying $\left.L_{\mathrm{IR}} / L(\mathrm{Br} \gamma) \approx 10^{5}\right]$.

Quantitatively it appears unlikely that the old stellar population can contribute significantly to the total luminosity emergent from grains within the Galactic center, however. The bulge $K$-band luminosity within the central 1 parsec radius is $\sim 1 \times 10^{6} L_{\odot}$ or somewhat less (Becklin \& Neugebauer 1968; 
Kent 1992). An elliptical galaxy or bulge population emits a spectral energy distribution with luminosity per frequency decade, $v F_{v}$, at $2000 \AA$ that is only $\sim 1 \%$ of that produced at 2 $\mu \mathrm{m}$ (see Bruzual \& Charlot 1993 for illustrative plots). With this scaling, we would expect only perhaps $\sim 10^{4} L_{\odot}$ of ultraviolet emission from the old stellar population, which is far less than the $\sim 10^{6} L_{\odot}$ emitted by grains in the same region. Radiative heating by a luminous young population is clearly implicated.

\section{CONSISTENCY BETWEEN INFRARED AND RADIO PROPERTIES}

\subsection{Radio Recombination Line and the Ionizing Continuum}

An additional probe of the ionization properties of Sgr A West is provided by radio recombination lines. For an assumed relative abundance of hydrogen and helium, comparison of the strengths of radiofrequency recombination-line intensities for the two elements provides an indication of whether the $\mathrm{H}^{+}$and $\mathrm{He}^{+}$zones are fully cospatial. The relative sizes of the two zones are in turn an indication of the hardness of the ionizing continuum (e.g., Osterbrock 1989). Observations of the $\mathrm{H} 76 \alpha$ and $\mathrm{He} 76 \alpha$ lines with resolution of $5^{\prime \prime} \times 10^{\prime \prime}$ $(\alpha \times \delta)$ were reported by Goss et al. (1990), who found a $2 \sigma$ upper limit to $\mathrm{He}^{+} / \mathrm{H}^{+}\left(\equiv y^{+}\right)$of 0.05 , measured over an unspecified portion of the nebula. Roberts \& Goss (1993) detected $\mathrm{He} 92 \alpha$ as well as $\mathrm{H} 92 \alpha$ at a location $6^{\prime \prime}$ east and $1^{\prime \prime}$ north of Sgr A*, and derived an estimate of $y^{+}=0.05 \pm 0.02$. They also report a $2 \sigma$ upper limit to $y^{+}$of 0.03 in a portion of the northern arm of the nebula. If the intrinsic helium abundance is $\sim 0.1$ that of hydrogen by number, a value of $y^{+} \leq 0.05$ would imply that the $\mathrm{He}^{+}$zone represents only approximately half or less of the volume of the $\mathrm{H}^{+}$zone, which would support the contention that the ionizing radiation field is moderately soft $\left(T_{*} \lesssim 38,000 \mathrm{~K}\right.$; Osterbrock 1989).

\subsection{Cloud Densities and Structure}

Analysis of the fine-structure emission-line spectrum $(\S 2.3)$ provides strong indications that much of the nebulosity in the central parsec features $n_{\mathrm{H}} \gtrsim 10^{5} \mathrm{~cm}^{-3}$, independent of the details of the ionizing continuum shape. Although the Galactic center shows strong evidence for recent star formation representing a substantial energy input, densities of this magnitude are large compared with those of normal $\mathrm{H}$ II regions, with the exception of ultracompact sources (e.g., Churchwell 1991). Relatively high densities for the nebular gas are not altogether unexpected, since molecular clouds surrounding the central parsec (i.e., the circumnuclear disk, or CND) are unusually dense $\left(\sim 10^{5} \mathrm{~cm}^{-3}\right)$ compared to their counterparts farther out in the Galaxy (e.g., Genzel 1989). The densest components within the CND have molecular densities comparable to the highest $n_{\mathrm{H}}\left(\sim 10^{7} \mathrm{~cm}^{-3}\right)$ employed in the models discussed in $\S 2.3$ (Jackson et al. 1993). High densities in the central parsec could arise from the increased depth of the gravitational potential at small radii, or possibly through compression in shocks. Dense plasma in the Galaxy center is of interest in light of direct indications that emission-line gas with similar densities is present in LINERs and more vigorous AGNs (e.g., Filippenko \& Halpern 1984; De Robertis \& Osterbrock 1984). Unusually high nebular densities may also play a fundamental role in causing some low-luminosity emission-line nuclei to appear " active" (Shields 1992).

Constraints on the cloud structure within the central parsec can be obtained from the nebular emission measure $E_{m}$, the integral of $n_{e}^{2}$ along line-of-sight distance. The spectral shape of radio free-free continuum emission and surface brightnesses of infrared recombination lines imply a total $E_{m}$ of a few times $10^{7}$ $\mathrm{cm}^{-6} \mathrm{pc}$ in the brighter parts of Sgr A West (e.g., Brown, Johnston, \& Lo 1981; Lacy et al. 1980; Table 1). If $n_{e} \approx 10^{5}$ $\mathrm{cm}^{-3}$ through most of this region, the nebular gas must then be clumped on scales of $\sim 0.01 \mathrm{pc}$ or less, with relatively high covering factor. The $E_{m}$ contributed by a single cloud is constrained by $U$ estimates obtained from model calculations presented in $\S 2.3$. By equating the total recombination rate within the cloud to the rate at which ionizing photons enter it, we can derive the expression

$$
E_{m}=\frac{U n_{e} c}{\alpha_{B}} \approx 2.9 \times 10^{4} U n_{e},
$$

with $E_{m}$ in units of $\mathrm{cm}^{-6} \mathrm{pc}$ for $n_{e}$ expressed in $\mathrm{cm}^{-3} \mathrm{pc}$ for $n_{e}$ expressed in $\mathrm{cm}^{-3}$; here $\alpha_{B}$ is the total hydrogen (Case B) recombination coefficient (taken from Hummer \& Storey 1987 for $T_{e}=7500 \mathrm{~K}$ ) and $c$ is the speed of light.

If the clouds are predominantly described by large $n_{\mathrm{H}}$ and small $U$, as in the three-component models presented in $\S 2.3$, $E_{m}$ contributed by a single cloud will be only of order $10^{5} \mathrm{~cm}^{-6}$ pc, and $\sim 100$ or somewhat more clouds must be present along the line of sight in order to produce the total inferred $E_{m}$. Such a scenario evidently requires a very high degree of clumping and a high covering factor of clumps to reproduce the observed surface brightness of emission from Sgr A West. If this is indeed the case, sources of ionizing radiation within this region probably also must be spatially distributed rather than highly concentrated. Alternatively, the high surface-brightness emission defining the Sgr A West "mini-spiral" might be defined primarily by locations of high-inclination plane-like structures ionized by external sources. For normal stars, the total inferred luminosity in ionizing photons within the central parsec will require up to a hundred $\mathrm{O}$ stars, depending on the characteristic $T_{*}$. The total number of hot stars could be larger if a fraction of their ionizing radiation escapes from the central parsec, eluding detection. A spatially dispersed ensemble of ionizing sources is thus not unreasonable.

A modest degree of clumping is well documented on many size scales for the surfaces of molecular clouds (e.g., Falgarone, Phillips, \& Walker 1992). The CND displays a high degree of clumping, with a volume-filling factor of only 5\%-10\% (Genzel 1989). The mechanisms responsible for sustaining such density inhomogeneities are poorly understood, although they are probably related to the nature of turbulence in these environments. If this is indeed the case, the high densities and significant clumping seen in the CND are at least qualitatively consistent with a substantial turbulent energy density observed in the same material (e.g., Güsten et al. 1987). Emission-line widths for ionized gas in the central parsec are supersonic (average FWHM $=50 \pm 10 \mathrm{~km} \mathrm{~s}^{-1}$ for $\mathrm{H} 92 \alpha$ in a $5^{\prime \prime} \times 10^{\prime \prime}$ beam; Roberts \& Goss 1993), implying that turbulence is also significant within the central nebula, and substantial clumping may be a natural expectation for this material. The large streaming velocities characterizing this region as well as potentially significant shearing motions could provide natural mechanisms for sustaining a high degree of turbulence and clumping of the nebular gas (Güsten et al. 1987). A high degree of density inhomogeneity is also characteristic of the emissionline regions of AGNs, which contain dense gas clouds with low filling factor (Osterbrock 1989). 
An alternative scenario requiring less exotic nebular structuring results if we assume the ionizing continuum is described by relatively cool $T_{*}$ and large $U$. As discussed in $\S 2.3 .1$, this scenario can reproduce many of the essential aspects of the Galactic center emission-line spectrum with the notable exception of $\mathrm{He}$ I $2.06 \mu \mathrm{m}$, which, however, may be modified from our predictions in realistic nebulae. If we assume that $n_{\mathrm{H}} \approx 10^{5}$ $\mathrm{cm}^{-3}$ and $U=0.01$, equation (1) implies that $E_{m}$ for a single cloud would be comparable to the total $E_{m}$ measured within the central parsec. Substantial clumping or special projection effects would then be unnecessary for explaining the measured surface brightness. The properties of this material would then be similar to that in ultracompact $H$ II regions, which feature similar $n_{\mathrm{H}}$ and $E_{m}$ (e.g., Churchwell 1991). Additional components would still be required in order to reproduce the overall spectrum, e.g., a lower density phase responsible for the [O III] lines, and a lower $U$ phase emitting the observed [O I].

\subsection{Gas-Phase Abundances and Dust}

An additional property that affects the nebular spectrum in Sgr A West is the abundance of heavy elements. Relatively high abundances in the inner stellar bulge of the Galaxy are well documented (Frogel 1989), and absorption-line spectra of stars in the Galactic center additionally suggest that the metallicity in this region may be approximately 2 times solar (Sellgren et al. 1987; K. Sellgren, private communication). Test calculations with model parameters as described in $\S 2.3$ indicate that the heavy-element fine-structure line strengths relative to $\mathrm{Br} \gamma$ scale approximately linearly with abundance for enrichment of this magnitude.

Grain depletion can reduce the gas-phase abundances of heavy elements and influence nebular behavior in several ways. In the low-ionization PDR zones, grain photoelectrons are expected to be the dominant source of heating (Tielens \& Hollenbach 1985). Grain heating can also become important at very high ionization parameter ( $\gtrsim 0.1$; Ferland 1994). Depletion of coolants will more generally affect the thermal balance and lead to higher equilibrium temperatures. While changes in nebular temperature can lead to large variations in the emitted optical forbidden-line spectrum, ground-state fine-structure transitions are insensitive to $T_{e}$ over the temperature range of interest. Grain depletion consequently affects the emitted infrared fine-structure spectrum primarily by changing the gasphase population of emitters; the depletion factor propagates nearly linearly into a reduction of emitted line flux, for a given density and $U$.

Grains can influence nebular behavior in one further way, by modifying the spectral energy distribution of the incident ionizing continuum. At sufficiently high $U$, the ionized column density can increase to a degree that Lyman continuum radiation becomes optically thick to absorption by dust (see Voit 1992 for a discussion). For grain properties and a dust-to-gas ratio representative of the general interstellar medium, the nebular structure begins to be modified by dust absorption at $U \gtrsim 10^{-2}$; this transition point scales inversely with the dustto-gas ratio. Specific absorption by interstellar dust is expected to peak at photon energies slightly greater than the hydrogen Lyman limit (Mathis 1986). As a result, the spectral energy distribution of ionizing radiation will be modified by selective dust absorption, with the result that the continuum becomes harder. Qualitatively this implies that stellar-photoionized nebulae will appear to be energized by a source that is hotter than the actual $T_{*}$ describing the star (e.g., Aannestad 1989).
For the scenarios considered here in which the Galactic center nebula is dominated by a low- $U$ component, this effect is unlikely to be important. If the nebula is dominated by a phase with $\log U \approx-2$, derived bounds for $T_{*}$ may require downward adjustment, although test calculations with plausible dust-to-gas ratios (i.e., up to a few times the interstellar average) imply that this effect does not introduce significant changes to $T_{*}$ constraints derived assuming a dust-free nebula. We note additionally that modification of the ionizing spectral energy distribution may be less than suggested in the preceding discussion if the grain population produces absorption that is gray compared to the interstellar mean, which appears to be the case, for example, in the molecular cloud OMC-1 (Bohlin \& Savage 1981; Baldwin et al. 1991), and possibly the nuclei of other galaxies (Laor \& Draine 1993; Lauer et al. 1993).

The overall metallicity of the Galactic center nebula is best estimated from ratios of lines that are responsive to abundance variation but relatively insensitive to parameters such as $n_{\mathrm{H}}, U$, and $T_{*}$. Elemental tracers that are unlikely to be strongly depleted onto grains are also obviously preferable. As noted previously, the fine-structure lines respond nearly linearly to gas-phase abundance. Inspection of Figures 1-3 implies that none of the lines is entirely insensitive to other parameters. We note, however, three ratios that come close to meeting these criteria, those being $[\mathrm{Ar} \mathrm{II}] / \mathrm{Br} \gamma,[\mathrm{N} \mathrm{III}] /[\mathrm{O} \mathrm{III}] 52 \mu \mathrm{m}$, and [Ne II]/Br $\gamma$. In the nebular models presented in $\S 2.3$, [Ar II]/ $\mathrm{Br} \gamma$ is underpredicted by a factor of $\sim 2$ when solar abundances are assumed, thus implying heavy-element enrichment at a level $\sim 2 Z_{\odot}$ (where $Z_{\odot}$ refers to solar abundances). The ratio $[\mathrm{N} \mathrm{III}] /[\mathrm{O} \mathrm{III}] 52 \mu \mathrm{m}$ is also underpredicted, by a factor of $\sim 3-5$. The $\mathrm{N} / \mathrm{O}$ abundance ratio is expected to vary approximately as $Z^{2}$, due to secondary nitrogen enrichment (see Hamann \& Ferland 1993 for a discussion), such that the $[\mathrm{N} \mathrm{III}] /[\mathrm{O}$ III $]$ ratio for the Galactic center may again imply $Z \approx 2 Z_{\odot}$. In the case of [Ne II]/Br $\gamma$, however, good agreement with the observations is obtained with solar abundances. The [Ne II] line is also probably one of the best-measured transitions for the central parsec (cf. Table 1). The evidence for abundances in excess of solar levels thus remains mixed. The accuracy of metallicity determinations for the Galactic center based on these line ratios is almost certainly limited by the inhomogeneous character of the observations. Derived abundances may also be clouded by errors in reddening corrections and uncertainties in atomic parameters (i.e., dielectronic recombination, $\S 2.3 .1$ ).

A consistency check on possible enrichment is available from radio recombination-line measurements of $T_{e}$. Roberts \& Goss (1993) report that their observations of the H92 $\alpha$ emission line and radio continuum are consistent with $T_{e}=7000$ $\pm 500 \mathrm{~K}$ throughout most of Sgr A West. A somewhat lower temperature of $\sim 5000 \mathrm{~K}$ is suggested by the ratios of Brackett lines relative to the radio continuum (Rieke et al. 1989; Maloney et al. 1992), although this estimate is subject to uncertainty due to extinction corrections. Average $T_{e}$ for the solarmetallicity plasma components described in $\S 2.3$ are listed in Table 4. The predicted $T_{e}$ values resulting from the $50,000 \mathrm{~K}$ ionizing continuum are in reasonable agreement with the radio estimate. The predicted $T_{e}$ for the $35,000 \mathrm{~K}$ continuum case are somewhat low, however; $T_{e}$ predicted for the dominant high $n_{\mathrm{H}}$ component is $6090 \mathrm{~K}$. Increasing the heavy-element abundance of the gas increases the nebular cooling efficiency, resulting in a decrease in equilibrium temperature. Table 4 also lists the average $T_{e}$ for the same components discussed earlier, 
TABLE 4

Dependence of Nebular Temperature on Abundances

\begin{tabular}{|c|c|c|c|c|c|c|}
\hline \multirow[b]{2}{*}{ PARAMETER } & \multicolumn{3}{|c|}{$T_{*}=35,000 \mathrm{~K}$} & \multicolumn{3}{|c|}{$T_{*}=50,000 \mathrm{~K}$} \\
\hline & 1 & $2^{a}$ & 3 & 1 & 2 & 3 \\
\hline $\log n_{\mathrm{H}} \ldots \ldots \ldots \ldots$ & 2.5 & 4 & 5 & 3 & 4 & 7 \\
\hline $\log U \ldots \ldots \ldots \ldots \ldots$ & -2 & -0.5 & -4.8 & -3.5 & -2.2 & -6 \\
\hline Weight $\ldots \ldots \ldots \ldots$ & 0.03 & 0.12 & 1 & 0.05 & 0.02 & 1 \\
\hline \multicolumn{7}{|c|}{ Nebular temperature $(\mathrm{K})$ : } \\
\hline$Z_{\odot}^{b} \ldots \ldots \ldots \ldots$ & 6390 & 6950 & 6090 & 7160 & 8170 & 6580 \\
\hline $2 \times Z_{\odot} \ldots \ldots \cdots$ & 4820 & 5740 & 4940 & 5410 & 6670 & 5720 \\
\hline $3 \times Z_{\odot} \cdots \cdots \cdots$ & 3720 & 5050 & 4330 & 4370 & 5870 & 5250 \\
\hline
\end{tabular}

a Truncated at hydrogen column density of $10^{20} \mathrm{~cm}^{-2}$.

b $Z_{\odot}=$ solar abundance of elements heavier than $\mathrm{He}$.

but with metallicity boosted to 2 and 3 times solar levels. For either scenario, enrichment to levels of only 2 times solar values may be inconsistent with the radio measurements of Sgr A West.

As noted above, inclusion of grains and associated depletion in the nebular calculations is expected to increase $T_{e}$ at a given $Z$. Calculations were repeated for components described by the $n_{\mathrm{H}}, U$, and $T_{*}$ listed in Table 4 , but with the gas-phase abundances of heavy elements depleted in proportions tabulated for the local interstellar medium by Cowie \& Songaila (1986). A corresponding population of interstellar graphite and silicate grains was also included, using the computational methods described in Baldwin et al. (1991). As the total $Z$ was increased, the ratio of grains/total metal abundance was assumed to remain constant. The results yield a mean increase in $T_{e}$ of $\sim 1200 \mathrm{~K}$ over the dust-free predictions given in Table 4 . If gas-phase abundances remain depleted in the interstellar proportions, and the dust/metals ratio is preserved, the measured $T_{e}$ would be consistent with total abundances of $1-2 \times Z_{\odot} ; Z$ as high as $3 Z_{\odot}$ would remain only marginally consistent.

To summarize, estimates of $T_{e}$ in Sgr A West are probably consistent with heavy-element enrichment of up to twice solar levels, but higher abundances might be difficult to reconcile. This upper bound may be relaxed if the temperature balance is significantly affected by heat sources other than photoionization, or if the radio measurements of $T_{e}$ are too high; the derived nebular temperatures could be excessive, for example, if high-velocity, low-amplitude wings of recombination emission exist and were not included in the line measurements. Estimates of abundance and nebular temperature are additionally sensitive to assumptions concerning depletion. Depletion onto grains is known to scale on average with interstellar gas density (Cowie \& Songalia 1986 and references therein). Given the apparent high density within the central parsec, this zone may be characterized by unusual and large depletions from the gas phase, which could then yield consistency between the observations and higher total heavy-element abundances.

\section{SGR A WEST IN PERSPECTIVE}

\subsection{Sgr $A$ West as an $\mathrm{H}$ II Region}

Sgr A West shows abundant evidence for recent star formation, and it is likely that the emission-line nebula in this region is energized primarily by young stars. As discussed earlier in this paper, the infrared fine-structure spectrum is consistent with photoionization by $\mathrm{O}$ stars, and radio-recombination-line determinations of $y^{+}$suggest that these stars have relatively modest effective temperatures. If gas in the central parsec has abundances that are 1-2 times solar, the presence of relatively cool stellar ionizing sources in this rather metal-rich environment is consistent with an apparent inverse correlation between ionizing continuum hardness (i.e., $T_{*}$ ) and enrichment observed in H II regions (e.g., McCall, Rybski, \& Shields 1985; McCaugh 1991).

In other aspects, Sgr A West does not represent a typical $\mathrm{H}$ II region, however. The emission-line spectrum of this region features some peculiarities when compared with other Galactic $\mathrm{H}$ II regions ( $\$ 2.3,3.3$ ); most notably, the [Ar II] line is unusually strong relative to other fine structure lines including both [S III] and [S IV] (see Herter et al. 1981, 1982; Pipher et al. 1984 for $\mathrm{H}$ II region comparisons). The ionizing luminosity within the region $\left(\sim 10^{50}\right.$ ionizing photons $\mathrm{s}^{-1}$; Lacy et al. 1980 ) is also relatively large. The nebular spectrum of the central parsec is most readily explained if much of the emitting plasma is described by unusually high $n_{\mathrm{H}}$ and possibly a low $U$ compared with the corresponding properties of typical $\mathrm{H}$ II regions. As discussed earlier, the molecular gas in this locale is similarly very dense and apparently highly clumped and turbulent, which may be related to unusually strong local streaming and shearing motions. Measurements of the gas kinematics in Sgr A West have been interpreted as reflecting the influence of a substantial central mass concentration $\left(\sim 10^{5}-10^{6} M_{\odot}\right.$; Lacy et al. 1991 and references therein), which is also not a standard constituent of $\mathrm{H}$ II regions. Properties of dust within the central parsec may be unusual $(\S 2.2)$, and the gas may also be somewhat metal rich $(\S 3.3)$. In light of these many distinctions, conclusions based on simple comparisons between Sgr A West and $\mathrm{H}$ II regions elsewhere in the Galaxy should be treated with caution.

\subsection{Sgr A West as a Galaxy Nucleus}

As discussed in the Introduction, comparison of the nebular properties of the nucleus of the Milky Way with those of other galaxy nuclei would be facilitated if the optical spectrum of the Galactic center were measurable. The stellar photoionization models constructed in $\S 2.3$ to explain the infrared spectrum can be used to predict the relative strength of optical emission lines, and the results of such an exercise are listed in Table 5. Solar abundances without depletion are again assumed in our initial calculations. It should be noted that the nebular parameters employed to reproduce the fine-structure spectrum are almost certainly not unique, and the predicted optical spectrum is thus only an approximate estimate. Comparison with classification criteria for emission-line objects (Baldwin et al. 1981; Veilleux \& Osterbrock 1987; Osterbrock, Tran, \& Veilleux 1992) indicates that the composite spectrum for photoionization with the $35,000 \mathrm{~K}$ stellar continuum satisfies the definition of LINERs based on the relative strength of the oxygen lines ([O III $] \lambda 5007:[\mathrm{O} \mathrm{II}] \lambda 3727:[\mathrm{O} \mathrm{I}]$ $\lambda 6300=1:>1:>0.3)$, yet features $[\mathrm{N} \mathrm{II}] \lambda 6583$ and $[\mathrm{S} \mathrm{II}]$ $\lambda 6724$ emission that is uncharacteristically weak for LINERs. The results for photoionization by the $35,000 \mathrm{~K}$ star are generally consistent with moderately low-excitation $\mathrm{H}$ II regions, except that [O I] $\lambda 6300$ is predicted to be much stronger than observed in such objects. The results for the $50,000 \mathrm{~K}$ stellar continuum cannot be described as LINER-like, and are also inconsistent with $\mathrm{H}$ II regions, due to the simultaneously low values of the $[\mathrm{O} \mathrm{III}] \lambda 5007 / \mathrm{H} \beta$ and $[\mathrm{N} \mathrm{II}] \lambda 6583 / \mathrm{H} \alpha$ intensity ratios. 
TABLE 5

Optical Spectrum Photolonization Predictions

\begin{tabular}{|c|c|c|c|c|c|c|c|c|}
\hline \multirow[b]{2}{*}{ PARAMETER } & \multicolumn{4}{|c|}{$T_{*}=35,000 \mathrm{~K}$} & \multicolumn{4}{|c|}{$T_{*}=50,000 \mathrm{~K}$} \\
\hline & 1 & $2^{a}$ & 3 & Composite & 1 & 2 & 3 & Composite \\
\hline $\begin{array}{l}\log n_{H} \ldots \ldots \ldots \ldots \ldots \ldots \ldots \ldots \\
\log U \ldots \ldots \ldots \ldots \ldots \ldots \ldots \ldots \ldots \\
\text { Weight } \ldots \ldots \ldots \ldots \ldots \ldots \ldots \ldots\end{array}$ & $\begin{aligned} & 2.5 \\
- & 2 \\
& 0.03\end{aligned}$ & $\begin{array}{c}4 \\
-0.5 \\
0.12\end{array}$ & $\begin{array}{c}5 \\
-4.8 \\
1\end{array}$ & & $\begin{array}{l}3 \\
-3.5 \\
0.05\end{array}$ & $\begin{array}{l}4 \\
-2.2 \\
0.02\end{array}$ & $\begin{array}{r}7 \\
-6 \\
1\end{array}$ & \\
\hline 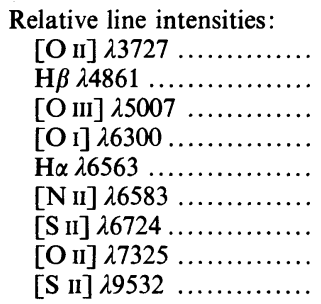 & $\begin{array}{l}2.0 \\
1 \\
0.02 \\
0 \\
2.7 \\
1.4 \\
0.17 \\
0.02 \\
1.1\end{array}$ & $\begin{array}{l}0.19 \\
1 \\
1.8 \\
0 \\
2.5 \\
0.02 \\
0 \\
0.01 \\
0.66\end{array}$ & $\begin{array}{l}0.15 \\
1 \\
0 \\
0.33 \\
2.6 \\
0.69 \\
0.22 \\
0.05 \\
0.04\end{array}$ & $\begin{array}{l}0.20 \\
1 \\
0.19 \\
0.29 \\
2.6 \\
0.64 \\
0.20 \\
0.04 \\
0.13\end{array}$ & $\begin{array}{l}3.3 \\
1 \\
0.81 \\
0.19 \\
2.7 \\
2.0 \\
1.4 \\
0.06 \\
0.87\end{array}$ & $\begin{array}{l}0.65 \\
1 \\
9.3 \\
0.04 \\
2.7 \\
0.47 \\
0.13 \\
0.08 \\
1.6\end{array}$ & $\begin{array}{l}0.01 \\
1 \\
0 \\
2.5 \\
2.7 \\
0.09 \\
0.05 \\
0.11 \\
0.02\end{array}$ & $\begin{array}{l}0.18 \\
1 \\
0.21 \\
2.3 \\
2.7 \\
0.19 \\
0.11 \\
0.11 \\
0.09\end{array}$ \\
\hline
\end{tabular}

a Truncated at hydrogen column density of $10^{20} \mathrm{~cm}^{-2}$.

Enrichment with $Z>Z_{\odot}$ will increase the population of heavy-element emitters but in so doing will also act to decrease nebular $T_{e}$, which will tend to diminish optical emission. However, grain depletion of selected coolants can offset in part the effect on $T_{e}$ of boosting global $Z$. Moreover, the elements responsible for optical emission employed in classification diagrams $(\mathrm{O}, \mathrm{N}, \mathrm{S})$ are not expected to be strongly depleted onto grains. As discussed previously $(\S 3.3)$, secondary production of nitrogen is also expected to produce a $Z^{2}$ dependence of $\mathrm{N}$ enrichment, which can lead to enhancement of [N $\mathrm{NI}]$ emission while producing only a small perturbation in total optical cooling established by the overall metallicity. Test calculations were consequently conducted with twice-solar abundances for all elements except nitrogen, four-times solar $\mathrm{N}$, and gas-phase abundances modified by standard interstellar grain depletion (with a constant grain/metals ratio), in order to examine the consequences for optical emission. For nebular components described by $U, n_{\mathrm{H}}$, and $T_{*}$ as listed in Table 5 , calculations with the $35,000 \mathrm{~K}$ stellar continuum resulted in [N II] emission that is consistent with LINER spectra $([\mathrm{N} \mathrm{II}] / \mathrm{H} \alpha=0.94)$, [S II] emission resembling that of $\mathrm{H}$ II regions ([S II]/ $\mathrm{H} \alpha=0.22$ ), and oxygen lines consistent with neither ([O III]: $[\mathrm{O} \mathrm{II}]:[\mathrm{O} \mathrm{I}]=0.53: 0.39: 0.57$, normalized to $\mathrm{H} \beta$ ). Calculations with the $50,000 \mathrm{~K}$ continuum resulted in increases in $[\mathrm{N} \mathrm{II}]$ and $[\mathrm{S} \mathrm{II}]$ emission and decreases in oxygen emission, but with no qualitative change in the overall character of predicted emission from that seen with undepleted solar abundances.

If the emission-line properties of Sgr A West can be reconciled with a dominant phase described by high $n_{\mathrm{H}}\left(\sim 10^{5} \mathrm{~cm}^{-3}\right)$, moderate $U(\sim 0.01)$, and soft incident radiation field, as discussed in $\S 2.3$, the predicted optical spectrum for undepleted solar abundances is again somewhat peculiar compared to that of external nuclei. Emission from nitrogen and oxygen relative to the Balmer lines is expected, not surprisingly, to be consistent with ratios for metal-rich $\mathrm{H}$ II regions ([N II $] / \mathrm{H} \alpha=0.5$, [O III] $/ \mathrm{H} \beta=0.008$ ). Due to the high $n_{e}$, however, predicted emission in the optical $[\mathrm{S} \mathrm{II}]$ lines $([\mathrm{S} \mathrm{II}] \lambda \lambda 6717,6731 /$ $\mathrm{H} \alpha=0.009$ ) is much less than in normal $\mathrm{H}$ II regions. This behavior contrasts sharply with that of $\mathrm{H}$ II nuclei, which show normal or enhanced [S II] emission compared to disk $\mathrm{H}$ II regions (Kennicutt, Keel, \& Blaha 1989). Emission of [O II] $\lambda 7325$ is also predicted to be very strong $([\mathrm{O} \mathrm{II}] / \mathrm{H} \alpha=0.2)$, in closer agreement with the behavior of Seyferts or LINERs than of $\mathrm{H}$ II nuclei (Osterbrock et al. 1992). Calculations with $2 Z_{\odot}$ and grains lead to LINER-like [N II] emission ([N II]/ $\mathrm{H} \alpha=1.3$ ), although [S II] remains very weak and the oxygen lines are again consistent with $\mathrm{H}$ II regions.

The disagreement between predicted optical emission for the Galactic center and measured line ratios for other galaxy nuclei is puzzling. While this disparity might be interpreted as casting doubt on the interpretation of the infrared spectrum described in $\S 2.3$, it should be remembered that substantial differences exist between metric apertures employed for the Galactic and extragalactic measurements. The present analysis of the Galactic center has focused on the properties of the central $\sim 1.2$ parsec, but existing optical spectroscopy of other galaxy nuclei has been obtained with apertures that project to size scales that are 1-2 orders of magnitude larger. To assess the importance of this aperture difference quantitatively, we note that $\sim 1.3 \times 10^{50}$ ionizing photons per second are absorbed within the central $\sim 2.4$ pc (Lacy et al. 1980), while $\sim 1.6 \times 10^{51}$ photoionizations $\mathrm{s}^{-1}$ occur within the central 200 pc (diameter, corrected to $R_{0}=8 \mathrm{kpc}$; Mezger \& Pauls 1979). Within metric apertures typical of extragalactic measurements, the central parsec will consequently contribute $\sim 10 \%$ of the total recombination luminosity; the corresponding largeaperture optical spectrum thus may differ significantly from the predictions in Table 5 .

The peculiarities of the predicted optical composite spectra in Table 5 can be largely traced to the very low $U$, high $n_{\mathrm{H}}$ component. Enlargement of the metric aperture to scales comparable to those of the extragalactic measurements would very likely lead to inclusion of less exotic nebular material characterized by higher $U$ and lower $n_{\mathrm{H}}$, which in turn would make the composite spectrum appear less anomalous. Hydrostatic equilibrium arguments would dictate that the gas in the center of the potential well is densest. Observations of LINERs also suggest empirically that the densest gas is concentrated to the smallest radii. Specially, LINERs exhibit a correlation between emission-line critical density and line width that implies that the densest material also exhibits the largest velocities (e.g., Filippenko \& Halpern 1984; Ho et al. 1993). If the kinematics of the nebular gas are determined primarily by orbital motion around a central mass concentration such as a black hole, gas velocity will increase with decreasing radius, and the empirical 
correlation then implies that the plasma density also increases toward smaller radii; small metric apertures will thus preferentially isolate dense gas.

Galaxies show a correlation between nuclear emission-line properties and Hubble type such that early-type galaxies show a strong tendency to feature LINER emission, while late-type objects contain H II nuclei (e.g., Heckman 1980; Terlevich, Melnick, \& Moles 1987). At the intermediate morphology of the Milky Way (Sb-Sbc; van der Kruit 1990), the presence of either nebular type may be equally probable. Sources of ionizing radiation that could power LINERs include AGN-like central engines generating power-law continua extending to X-rays (Ferland \& Netzer 1983; Halpern \& Steiner 1983), extreme Wolf-Rayet stars (Terlevich \& Melnick 1985), and relatively hot, yet normal main-sequence O stars (Filippenko \& Terlevich 1992; Shields 1992).

The absence of strong source of hard X-rays within the central parsec implies that photoionization by a weak, classical AGN is unlikely. The presence of extreme WO and WC stars would be consistent with weak X-ray emission, and direct observations of the stellar population in the central parsec do not exclude these objects. High-mass stars that give rise to extreme W-R sources evolve at approximately constant bolometric luminosity. In the $\mathrm{W}-\mathrm{R}$ phase, these objects are exceedingly hot $\left(\sim 10^{5} \mathrm{~K}\right)$ yet less luminous at $2 \mu \mathrm{m}$ than their main-sequence forebears, so that such stars would remain near or as much as 2 magnitudes fainter than detection thresholds in existing deep K-band imaging ( $\sim 14.5 \mathrm{mag}$; Herbst, Beckwith, \& Shure 1993). The presence of recent star formation and the lack of diagnostics of $T_{*}$ afforded by the infrared continuum and emission-line spectrum would also be consistent with the presence of massive, hot stars on the main sequence or in an extreme $\mathrm{W}-\mathrm{R}$ phase. Hot $\mathrm{O}$ or $\mathrm{W}-\mathrm{R}$ stars are likely excluded, however, by limits on $T_{*}$ implied by the radio recombination-line measurements of $y^{+}(\S 3.1)$. The Milky Way thus probably presents an $\mathrm{H}$ II nucleus to observers outside our Galaxy.

While the Milky Way does not clearly evince the presence of an active nucleus through its emission-line intensities, the presence of a massive, central black hole such as may drive a classical AGN may be indicated by gaseous and stellar kinematic tracers (e.g., Lacy et al. 1991; McGinn et al. 1989). An identification of such a black hole with the unusual radio source $\mathrm{Sgr} \mathrm{A}^{*}$ is often suggested. If $\mathrm{Sgr} \mathrm{A}^{*}$ is indeed a black hole, it is unlikely to be important for photoionizing the Galactic center nebula unless its spectral energy distribution is very strongly peaked at extreme ultraviolet energies; the nature of any such radiation is additionally restricted by estimates of $y^{+}$, as discussed above for stellar sources. The results of the present work suggesting that nebular gas in the central parsec is relatively dense may furthermore lend some support to an alternative view that $\mathrm{Sgr} \mathbf{A}^{*}$ is actually a pulsar embedded in a dense medium, resulting in the anomalous radio properties of this object (Phinney 1989 and references therein). Shrinking upper limits to the proper motion of $\mathrm{Sgr} \mathrm{A}^{*}$ increasingly suggest that this object is more massive than a neutron star, however (Backer \& Sramek 1987; D. C. Backer, private communication).

\section{CONCLUSIONS}

This work describes an attempt to understand the implications of the composite infrared spectrum for the properties of the nebular gas and ionizing continuum radiation in the
Galactic center. The results indicate that the emitted spectrum is more sensitive in many aspects to ionization parameter and cloud density than to details of the ionizing spectral energy distribution. The often-quoted limit of $35,000 \mathrm{~K}$ for the effective temperature of ionizing stars in this region is not strongly supported on the basis of the infrared nebular spectrum if a wider range of $U$ and $n_{\mathrm{H}}$ than characterizes normal $\mathrm{H}$ II regions is considered. In particular, harder continua such as are generated by early $\mathrm{O}$ stars or represented by an extremeultraviolet power law cannot be excluded on the basis of the infrared spectrum. Independent evidence that the ionizing radiation field is relatively soft in Sgr A West is provided by measurements of $y^{+}$from radio recombination-line measurements, which implicate a smaller fractional ionization of $\mathrm{He}$ than of $\mathbf{H}$. The effective temperature of stellar sources that might be responsible for photoionizing most of this nebulosity is then $T_{*}<40,000 \mathrm{~K}$. A lower bound of $T_{*}>30,000 \mathrm{~K}$ is additionally provided by the strength of infrared lines of intermediate ionization (e.g., [O III], [N III]).

Regardless of the details of the ionizing spectral energy distribution, the infrared spectrum from the central parsec provides strong evidence that this nebula is quite dense $\left(n_{\mathrm{H}} \gtrsim 10^{5}\right.$ $\mathrm{cm}^{-3}$ ). Relatively high densities are directly indicated by measurements in some locations of density-sensitive line ratios (e.g., DePoy 1992). Several other aspects of the fine-structure spectrum, notably the strength of [Ar II] emission and (especially) the weakness of sulfur lines, are most readily understood if much of the ionized plasma is unusually dense compared to common disk $\mathrm{H}$ II regions.

Consistency with the emission-line spectrum of Sgr A West is best obtained in the present photoionization calculations if most of the radiating plasma is described by rather low ionization parameter. The observed surface brightness of emission would then require a substantial degree of clumping and superposition of clouds along the line of sight. Substantial clumping is observed in circumnuclear molecular material, and might be sustained in the central parsec by unusual fluid-dynamical properties in this zone. Less clumping is required if most of the gas is described by a somewhat larger $U$, which can be reconciled with most of the observed emission-line ratios.

The strengths of infrared emission lines provide mixed evidence for enrichment above solar levels, with argon and nitrogen emission consistent with $Z \approx 2 Z_{\odot}$, but neon emission implying $Z \approx Z_{\odot}$. The reliability of these estimates is limited by the inhomogeneity of published emission-line measurements. Equilibrium temperature estimates for nebulae with $Z=1-2 \times Z_{\odot}$ are consistent with $T_{e}$ estimates derived from radio recombination line-to-continuum ratios.

Simple estimates of the optical emission-line spectrum of the central parsec appear somewhat anomalous in comparison with other galaxy nuclei, although such differences may be due to the much larger metric apertures employed in extragalactic observations. The peculiarities of the predicted optical spectrum are largely traceable to the dense medium required to reproduce the infrared line emission. This result is consistent with expectations from extragalactic studies that smaller metric apertures will isolate characteristically denser gas within galaxy nuclei. When observed in a larger aperture typical of extragalactic studies, the Milky Way probably exhibits an $\mathrm{H}$ II nucleus to external observers.

Sgr A West may differ from average $\mathrm{H}$ II regions on the basis of its ionization state, density, enrichment, dust content, and velocity field, and consequently some circumspection is 
required when drawing conclusions from comparisons of the Galactic center with other disk nebulae. In recent years observational study of ionized gas in the Galactic center has tended to emphasize kinematic features, which is understandable given the interest in whether this region hosts a massive black hole. However, given the unusual properties of diffuse matter in the central parsec and the significance of this environment as the nearest galaxy nucleus, additional attention to the ionization properties of this material is desirable. More extensive observations of the infrared fine-structure lines with improved spatial resolution would help to elucidate the structural char- acteristics and underlying sources of energy for this important nebula. Large-aperture measurements are also necessary for meaningful comparisons with extragalactic nuclei.

We thank D. DePoy, M. Elitzur, J. Jackson, R. Kennicutt, J. Lacy, K. Sellgren, and F. Yusef-Zadeh for illuminating discussions and comments concerning this work. The comments of the referee, R. Genzel, both before and after submission of this manuscript, were particularly helpful. This research was supported by NSF grant AST 90-19692.

\section{REFERENCES}

Aannestad, P. A. 1989, ApJ, 338, 162

Aitken, D. K., Griffiths, J., Jones, B., \& Penman, J. M. 1976, MNRAS, 174, 41P

Aitken, D. K., Jones, B., \& Penman, J. M. 1974, MNRAS, 169, 35P

Ali, B., Blum, R. D., Bumgardner, T. E., Cranmer, S. R., Ferland, G. J., Haefner, R. I., \& Tiede, G. P. 1991, PASP, 103, 1182

Backer, D. C., \& Sramek, R. A. 1987, in The Galactic Center, ed. D. C. Backer (New York: AIP), 163

Baldwin, J. A., Ferland, G. J., Martin, P. G., Corbin, M. R., Cota, S. A., Peterson, B. M., \& Slettebak, A. 1991, ApJ, 374, 580

Baldwin, J. A., Phillips, M. M., \& Terlevich, R. 1981, PASP, 93, 5

Bally, J., Joyce, R. R., \& Scoville, N. Z. 1979, ApJ, 229, 917

Becklin, E. E., \& Neugebauer, G. 1968, ApJ, 151, 145

Berrington, K. A., \& Kingston, A. E. 1987, J. Phys. B, 20, 6631

Bohlin, R. C., \& Savage, B. D. 1981, ApJ, 249, 109

Brown, R. L., Johnston, K. J., \& Lo, K. Y. 1981, ApJ, 250, 155

Bruzual A., G., \& Charlot, S. 1993, ApJ, 405, 538

Burstein, D., Bertola, F., Buson, L. M., Faber, S. M., \& Lauer, T. R. 1988, ApJ, 328,440

Churchwell, E. 1991, in The Physics of Star Formation and Early Stellar Evolution, ed. C. J. Lada \& N. D. Kylafis (Dordrecht: Kluwer), 221

Cota, S. A. 1987, Ph.D. dissertation, Ohio State Univ.

Cowie, L. L., \& Songaila, A. 1986, ARA\&A, 24, 499

Dain, F. W., Gull, G. E., Melnick, G., Harwit, M., \& Ward, D. B. 1978, ApJ, 221, L17

Davidson, J. A., Werner, M. W., Wu, X., Lester, D. F., Harvey, P. M., Joy, M., \& Morris, M. 1992, ApJ, 387, 189

Davidson, K., \& Netzer, H. 1979, Rev. Mod. Phys., 51, 715

DePoy, D. L. 1992, ApJ, 398, 512

De Robertis, M. M., \& Osterbrock, D. E. 1984, ApJ, 286, 171

Falgarone, E., Phillips, T. G., \& Walker, C. 1991, ApJ, 378, 186

Ferguson, H. C., et al. 1991, ApJ, 382, L69

Ferguson, H. C., \& Davidsen, A. F. 1993, ApJ, 408, 92

Ferland, G. J. 1993, Univ. Kentucky Dept. of Physics and Astronomy Internal Rep.

Rep. 1994, in The Nearest Active Galaxies, ed. J. Beckman (Madrid: CSIC), in press

Ferland, G. J., \& Netzer, H. 1983, ApJ, 264, 105

Ferland, G. J., \& Rees, M. J. 1988, ApJ, 332, 141

Filippenko, A. V., ed. 1992, Relationships between Active Galactic Nuclei and Starburst Galaxies (San Francisco: Astronomical Society of the Pacific)

Filippenko, A. V., \& Halpern, J. P. 1984, ApJ, 285, 458

Filippenko, A. V., \& Sargent, W. L. W. 1985, ApJS, 57, 503

Filippenko, A. V., \& Terlevich, R. 1992, ApJ, 399, 79

Frogel, J. A. 1989, in IAU Symp. 136, The Center of the Galaxy, ed. M. Morris (Dordrecht: Kluwer), 55

Geballe, T. R., Krisciunas, K., Lee, T. J., Gatley, I., Wade, R., Duncan, W. D., Garden, R., \& Becklin, E. E. 1984, ApJ, 284, 118

Genzel, R. 1989, in IAU Symp. 136, The Center of the Galaxy, ed. M. Morris (Dordrecht: Kluwer), 393

Genzel, R., Stacey, G. J., Harris, A. I., Townes, C. H., Geis, N., Graf, U. U., Poglitsch, A., \& Stutzki, J. 1990, ApJ, 356, 160

Genzel, R., \& Townes, C. H. 1987, ARA\&A, 25, 377

Genzel, R., Watson, D. M., Crawford, M. K., \& Townes, C. H. 1985, ApJ, 297, 766

Genzel, R., Watson, D. M., Townes, C. H., Dinerstein, H. L., Hollenbach, D., Lester, D. F., Werner, M., \& Storey, J. W. V. 1984, ApJ, 276, 551

Gross, W. M., van Gorkom, J. H., Roberts, D. A., \& Leahy, J. P. 1990, Radio, Recombination Lines: 25 Years of Investigation, ed. M. A. Gordon \& R. L. Sorochenko (Dordrecht: Kluwer), 249

Graf, P., Herter, T., Gull, G. E., \& Houck, J. R. 1988, ApJ, 330, 803

Greggio, L., \& Renzini, A. 1990, ApJ, 364, 35

Grevesse, N., \& Anders, E. 1989, in Cosmic Abundances of Matter, ed. C. J. Waddington (New York: AIP), 1

Güsten, R., Genzel, R., Wright, M. C. H., Jaffe, D. T., Stutzki, J., \& Harris, A. I. 1987, ApJ, 318, 124

Halpern, J. P., \& Steiner, J. E. 1983, ApJ, 269, L37

Hamann, F., \& Ferland, G. J. 1993, ApJ, 418, 11

Heckman, T. M. 1980, A\&A, 87, 152
Herbst, T. M., Beckwith, S. V. W., \& Shure, M. 1993, ApJ, 411, L21

Herter, T., Briotta, D. A., Gull, G. E., Shure, M. A., \& Houck, J. R. 1983, ApJ, 267, L37

Herter, T., Helfer, H. L., Pipher, J. L., Briotta, D. A., Forrest, W. J., Houck,

J. R., Rudy, R. J., \& Willner, S. P. 1982, ApJ, 262, 153

Herter, T., et al. 1981, ApJ, 250, 186

Herter, T., Houck, J. R., Graf, P., \& Gull, G. E. 1986, ApJ, 309, L13

Herter, T., Houck, J. R., Shure, M., Gull, G. E., \& Graf, P. 1984, ApJ, 287, L15

Ho, L. C., Filippenko, A. V., \& Sargent, W. L. W. 1993, ApJ, 417, 63

Hollenbach, D., \& McKee, C. F. 1989, ApJ, 342, 306

Hummer, D. G. 1968, MNRAS, 138, 73

. 1981, Journ. Quant. Spectr. Rad. Transf., 26, 187

Hummer, D. G., \& Kunasz, P. B. 1980, ApJ, 236, 609

Hummer, D. G., \& Storey, P. J. 1987, MNRAS, 224, 801

Jackson, J. M., Geis, N., Genzel, R., Harris, A. I., Madden, S., Poglitsch, A.,

Stacey, G. J., \& Townes, C. H. 1993, ApJ, 402, 173

Kennicutt, R. C., Keel, W. C., \& Blaha, C. A. 1989, AJ, 97, 1022

Kent, S. M. 1992, ApJ, 387, 181

Krabbe, A., Genzel, R., Drapatz, S., \& Rotaciuc, V. 1991, ApJ, 382, L19

Kurucz, R. L. 1979, ApJS, 40, 1

Lacy, J. H., Achtermann, J. M., \& Serabyn, E. 1991, ApJ, 380, L71

Lacy, J. H., Baas, F., Townes, C. H., \& Geballe, T. R. 1979, ApJ, 227, L17

Lacy, J. H., Townes, C. H., Geballe, T. R., \& Hollenbach, D. J. 1980, ApJ, 241, 132

Laor, A., \& Draine, B. T. 1993, ApJ, 402, 425

Lauer, T. R., et al. 1993, ApJ, 106, 1436

Lester, D. F., Bregman, J. D., Witteborn, F. C., Rank, D. M., \& Dinerstein, H. L. 1981a, ApJ, 248, 524

Lester, D. F., Dinerstein, H. L., Werner, M. W., Watson, D. M., Genzel, R., \&

Storey, J. W. V. 1987, ApJ, 320, 573

Lester, D. F., Werner, M. W., Storey, J. W. V., Watson, D. M., \& Townes, C. H. 1981b, ApJ, 248, L109

Maloney, P. R., Hollenbach, D. J., \& Townes, C. H. 1992, ApJ, 401, 559

Mathis, J. S. 1986, PASP, 98, 995

1990, ARA\&A, 28, 37

Matteson, J. L. 1982, in The Galactic Center, ed. G. R. Riegler \& R. D. Blandford (New York: AIP), 109

McCall, M. L., Rybski, P. M., \& Shields, G. A. 1985, ApJS, 57, 1

McCarthy, J. F., Forrest, W. J., Briotta, D. A., \& Houck, J. R. 1980, ApJ, 242, 965

McGaugh, S. S. 1991, ApJ, 380, 140

McGinn, M. T., Sellgren, K., Becklin, E. E., \& Hall, D. N. B. 1989, ApJ, 338 , 824

Meixner, M., \& Tielens, A. G. G. M. 1993, ApJ, 405, 216

Mezger, P. G., \& Pauls, T. 1979, in IAU Symp. 84, The Large-Scale Character-

istics of the Galaxy, ed. W. B. Burton (Dordrecht: Reidel), 357

Nahar, S. N., \& Pradhan, A. K. 1992, ApJ, 397, 719

Netzer, H., \& Laor, A. 1993, ApJ, 404, L51

Neugebauer, G., Becklin, E. E., Matthews, K., \& Wynn-Williams, C. G. 1978 ApJ, 220, 149

Nussbaumer, H., \& Storey, P. J. 1983, A\&A, 126, 75

Osterbrock, D. E. 1989, Astrophysics of Gaseous Nebulae and Active Galactic Nuclei (Mill Valley: University Science)

Osterbrock, D. E., Tran, H. D., \& Veilleux, S. 1992, ApJ, 389, 196

Pedlar, A., Anantharamaiah, K. R., Ekers, R. D., Goss, W. M., van Gorkom, J. H., Schwarz, U. J., \& Zhao, J. 1989, ApJ, 342, 769

Phinney, E. S. 1989, in IAU Symp. 136, The Center of the Galaxy, ed. M. Morris (Dordrecht: Kluwer), 543

Pipher, J. L., Helfer, H. L., Herter, T., Briotta, D. A., Houck, J. R., Willner, S P., \& Jones, B. 1984, ApJ, 285, 174

Poglitsch, A., Stacey, G. J., Geis, N., Haggerty, M., Jackson, J., Rumitz, M. Genzel, R., \& Townes, C. H. 1991, ApJ, 374, L33

Rich, R. M. 1988, AJ, 95, 828

Rieke, G. H., Rieke, M. J., \& Paul, A. E. 1989, ApJ, 336, 752

Roberts, D. A., \& Goss, W. M. 1993, ApJS, 86, 133

Rubin, R. H. 1989, ApJS, 69, 897

Sellgren, K., Hall, D. N. B., Kleinmann, S. G., \& Scoville, N. Z. 1987, ApJ, 317, 881 
Serabyn, E., \& Lacy, J. H. 1985, ApJ, 293, 445

Shields, J. C. 1991, AJ, 102, 1314

1992, ApJ, 399, L27

. 1993, ApJ, 419, 181

Shull, J. M., \& McKee, C. F. 1979, ApJ, 227, 131

Spinoglio, L. \& Malkan, M. A. 1992, ApJ, 399, 504

- Storey, J. W. V. \& Allen, D. A. 1983, MNRAS, 204, 1153

Terlevich, R., \& Melnick, J. 1985, MNRAS, 213, 841

Terlevich, R., Melnick, J., \& Moles, M. 1987, in IAU Symp. 121, Observational Evidence for Activity in Galaxies, ed. Y. Ye. Khachikyan, K. J. Fricke, \& J. Melnick (Dordrecht: Reidel), 499

Tielens, A. G. G. M.. \& Hollenbach, D. 1985, ApJ, 291, 722

van der Kruit, P. C. 1990, in The Milky Way as a Galaxy, ed. R. Buser \& I. King (Mill Valley: University Science), 331

Veilleux, S., \& Osterbrock, D. E. 1987, ApJS, 63, 295

Voit, G. M. 1992, ApJ, 399, 495

Wade, R., Geballe, T. R., Krisciunas, K., Gatley, I., \& Bird, M. C. 1987, ApJ, 320,570
Watson, D. M., Crawford, M. K., Genzel, R., \& Townes, C. H. 1985, in preparation (cited in Genzel et al. 1985)

Watson, D. M., Storey, J. W. V., Townes, C. H., \& Haller, E. E. 1980, ApJ, 241, L43

Watson, M. G., Willingale, R., Grindlay, J. E., \& Hertz, P. 1981, ApJ, 250, 142

Wiese, W. L., Smith, M. W., \& Glennon, B. M. 1966, Atomic Transition Probabilities, Vol. 1 (Washington, DC: NBS)

Willner, S. P. 1978, ApJ, 219, 870

Willner, S. P., \& Pipher, J. L. 1983, ApJ, 265, 760

Willner, S. P., Russell, R. W., Puetter, R. C., Soifer, B. T., \& Harvey, P. M. 1979, ApJ, 229, L65

Wolfire, M. G., Tielens, A. G. G. M. \& Hollenbach, D. 1990, ApJ, 358, 116

Wollman, E. R., Geballe, T. R., Lacy, J. H., Townes, C. H., \& Rank, D. M. 1976, ApJ, 205, L5

. 1977, ApJ, 218, L103

Wollman, E. R., Smith, H. A., \& Larson, H. P. 1982, ApJ, 258, 506

Wynn-Williams, C. G., \& Becklin, E. E. 1974, PASP, 86, 5 